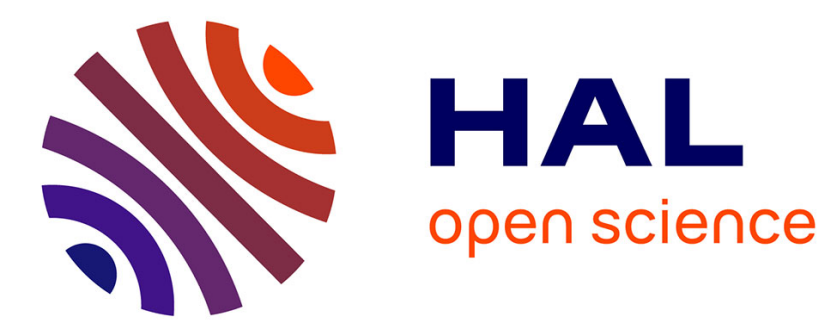

\title{
On the Uncertainty Quantification of the Quality Factor of Reverberation Chambers
}

\author{
Luk R. Arnaut, Philippe Besnier, Jérôme Sol, Mihai Ionut Andries
}

\section{To cite this version:}

Luk R. Arnaut, Philippe Besnier, Jérôme Sol, Mihai Ionut Andries. On the Uncertainty Quantification of the Quality Factor of Reverberation Chambers. IEEE Transactions on Electromagnetic Compatibility, 2019, 61 (3), pp.823-832. 10.1109/TEMC.2018.2839345 . hal-01909391

\section{HAL Id: hal-01909391 \\ https://hal.science/hal-01909391}

Submitted on 31 Oct 2018

HAL is a multi-disciplinary open access archive for the deposit and dissemination of scientific research documents, whether they are published or not. The documents may come from teaching and research institutions in France or abroad, or from public or private research centers.
L'archive ouverte pluridisciplinaire HAL, est destinée au dépôt et à la diffusion de documents scientifiques de niveau recherche, publiés ou non, émanant des établissements d'enseignement et de recherche français ou étrangers, des laboratoires publics ou privés. 


\section{On the Uncertainty Quantification of the Quality Factor of Reverberation Chambers}

ARNAUT, LR; Besnier, P; Sol, J; Andries, MI

(C) 2018 IEEE. Personal use of this material is permitted. Permission from IEEE must be obtained for all other uses, in any current or future media, including reprinting/republishing this material for advertising or promotional purposes, creating new collective works, for resale or redistribution to servers or lists, or reuse of any copyrighted component of this work in other works.

For additional information about this publication click this link.

http://qmro.qmul.ac.uk/xmlui/handle/123456789/44205

Information about this research object was correct at the time of download; we occasionally make corrections to records, please therefore check the published record when citing. For more information contact scholarlycommunications@qmul.ac.uk 


\title{
On the Uncertainty Quantification of the Quality Factor of Reverberation Chambers
}

\author{
Luk R. Arnaut, Philippe Besnier, Jérôme Sol, Mihai I. Andries
}

\begin{abstract}
Experimental characteristics of the normalized standard deviation and confidence intervals of the quality factor $(Q)$ of a mode-stirred reverberation chamber are compared with theoretical results. Fully empirical (model-free) statistics based on spectrally averaged S-parameter data show good agreement with semi-analytical estimates obtained from the theoretical FisherSnedecor distribution of $Q$ with empirical distribution parameter $M$. For increasing frequency, the distribution of the boundaries of the sample confidence intervals rapidly tends toward Gauss normality. The location of the boundaries agrees closely with theory, while their spread reduces upon spectral averaging. Distributions and confidence intervals for the maximum and minimum $Q$ are also obtained.
\end{abstract}

Index Terms-mode-stirred reverberation chambers, quality factor, inverse uncertainty quantification

\section{INTRODUCTION}

In previous work, theoretical statistics [1] and probability density functions (PDFs) [2] of the quality factor $(Q)$ for a mode-tuned or mode-stirred reverberation chamber (MT/MSRC) were derived. The full probabilistic characterization of $Q$ extends the notion of the effective (or composite) quality factor $Q_{\text {eff }}$ [1]-[6], which merely provides an averaged value in some sense [2]. In [7], an experimental method was presented for evaluating the PDF $f_{Q}(q)$ based on S-parameter measurements. In particular, the distributions of the maximum and minimum $Q$ are of interest in EMC testing for emissions, immunity, sensitivity, and fading in signal propagating.

As a system parameter, $Q$ affects the uncertainty of output fields and currents in MT/MSRCs. Therefore, as an uncertainty source, $f_{Q}(q)$ and its associated confidence intervals (CIs) are fundamental in the uncertainty quantification (UQ) and propagation (UP). Traditionally, (forward) UP relies on $a$ priori chosen, modelled, or otherwise estimated input PDFs for the uncertainty sources as inputs to UQ [8]. Inverse UP to $Q$ allows for validation and/or refinements to $f_{Q}(q)$ to be made, thus increasing the accuracy of forward UQ in turn [9].

From the perspective of MT/MSRCs as cavity resonators, the ratio of the stored and dissipated energies for noninteracting (isolated) modes $m n p$ is equivalent to their scaled resonance width, $Q_{m n p}=\Delta \omega_{m n p} / \omega_{m n p}$, where $\Delta \omega_{m n p}$ obeys the celebrated Porter-Thomas $\chi^{2}$ distribution [10]. In the overmoded regime of moderate or high modal overlap, however, modal coupling affects the width distribution and one must take recourse to the original macroscopic definition, viz., $Q(\omega)=U /\left(P_{\mathrm{d}} / \omega\right)$.

In this paper, experimental and theoretical results for the normalized standard deviation $\nu_{Q}=\sigma_{Q} / \mu_{Q}$, CI boundaries $\xi^{ \pm}$and extreme values of $Q$ are investigated. Fully empirical values are compared with semi-analytic values obtained based on ideal theoretical characteristics. Fully empirical estimates follow directly from measured data as sample statistics, without recourse to the PDF model, and are solely based on spectral averaging of S-parameter data followed by (mechanical) stir averaging. The semi-analytic statistics are obtained from the theoretical Fisher-Snedecor (F-S) PDF $f_{Q}(q)$ [2], whose distribution parameter $M$ is estimated empirically from the mean and standard deviation of the data. Since the theoretical results involve neither spectral averaging nor modelling of $M$, an agreement between both methods demonstrates consistency between the F-S model and the evaluation method.

For economy of notation, Greek symbols $\mu_{Q}, \sigma_{Q}, \nu_{Q}$, $\xi_{(Q)}^{ \pm}$denote both ensemble and sample statistics alike; their distinction will be clear from the context. The parameters $M$, $N, m$ and $n$ all represent deterministic quantities.

\section{Fluctuation of Transmission in MT/MSRCs}

Consider an unstirred cavity of interior volume $V$, surface area $S$ and skin depth $\delta_{\mathrm{w}}$ for its walls. For point-to-point transmission between two antennas inside the cavity, $Q$ represents an amplification factor between the transmitted power $P_{\mathrm{T}}$ and the spatially averaged received power $\left\langle P_{\mathrm{R}}\right\rangle_{V}$, i.e., [3]

$$
\left\langle P_{\mathrm{R}}\right\rangle_{V}=\frac{\lambda^{3} Q}{16 \pi^{2} V} P_{\mathrm{T}} .
$$

This indicates that $\left\langle P_{\mathrm{R}}\right\rangle_{V}$ is proportional to $Q$, which compensates for the inversely proportional attenuation of power density (space loss) when the electrical size of the cavity $V / \lambda^{3}$ increases. If wall absorption is the dominant loss mechanism then $Q \propto V /\left(\delta_{\mathrm{w}} S\right)$, whence the average insertion loss for $\lambda \rightarrow 0$ is then independently of $V$, viz.,

$$
\frac{\left\langle P_{\mathrm{R}}\right\rangle_{V}}{P_{\mathrm{T}}} \propto \frac{1}{f^{5 / 2} S}
$$

In comparison, for line-of-sight transmission in free space between aligned and polarization matched antennas separated by a distance $r$, the path loss is given by the Friis formula, $P_{\mathrm{R}} / P_{\mathrm{T}}=G_{\mathrm{T}} G_{\mathrm{R}} \lambda^{2} /\left(4 \pi r^{2}\right)$, whence $P_{\mathrm{R}} / P_{\mathrm{T}} \propto(f r)^{-2}$ provided that the antenna gains $G_{\mathrm{T}, \mathrm{R}}$ are independent of frequency. Since the ratio between this free-space path loss and (2) is proportional to $\sqrt{f}$, this indicates different dispersive characteristics between both environments. Consequently, non$\mathrm{CW}$ excitation and the spectral aggregation method for evaluating $f_{Q}(q)$ (cf. Sec. III-A) benefit from keeping the bandwidth small. This dispersion is relevant when using MT/MSRCs for emulating multipath propagation of wideband signals. Note 
that (2) and the $\sqrt{f}$-dichotomy only apply to the spatial average of the insertion loss, rather than to its point-to-point fluctuations.

Equation (1) assumes that $Q$ and $P_{\mathrm{T}}$ do not vary inside a given static cavity. ${ }^{1}$ A spatial average of the stored energy can be defined as $U=V \cdot\langle U\rangle_{V}$. In practice, $Q$ and $P_{\mathrm{T}}$ may fluctuate considerably during the stir process. This prompts a reconsideration of (1) for instantaneous (sampled) $P_{\mathrm{R}}, P_{\mathrm{T}}$ and $Q$, i.e., for each stir state $\tau$. These then represent quasi-static random EM quantities, i.e.,

$$
\left\langle P_{\mathrm{R}}(\tau)\right\rangle_{V}=\frac{\lambda^{3} Q(\tau)}{16 \pi^{2} V} P_{\mathrm{T}}(\tau)
$$

before applying ensemble averaging, to be denoted by $\langle\cdot\rangle$. The expression (3) serves as an implicit definition of a fluctuating $Q(\tau)$, where mechanical stirring generates a change of geometry and a consequent change of $Q$ (cf., e.g., [12, eq. (8.96)]).

\section{Measurement And Estimation Methods}

\section{A. Spectral Aggregation and Averaging}

Details of the experimental set-up and method for evaluating $f_{Q}(q)$ were given in [7, sec. IV.A], [11, sec. V.A]. Briefly, triplets of complex S-parameters $\left(S_{21}, S_{11}, S_{22}\right)$ are measured at a set of discrete frequencies $f_{i}\left(i=1, \ldots, N_{f}\right)$ and stir states $\tau_{j}\left(j=1, \ldots, N_{s}\right)$. These are used with (5) and (6) in spectral averaging across a bandwidth $\Delta f$ to be specified, in order to estimate spatial aggregates of the stored and dissipated energies. Spectral averaging offers an efficient alternative to spatial scanning [13] that is expected to be valid at sufficiently high frequencies, provided that strict-sense spatio-spectral ergodicity with respect to $f_{Q}(q)$ holds. Finally, probabilities and statistics of $Q$ are estimated based on ensemble averaging across all $\tau_{j}$.

Specifically, at $\omega_{i}=2 \pi f_{i}=2 \pi \mathrm{c} / \lambda_{i}$ and $\tau_{j}$ (cf. [7] for definitions of symbols),

$$
Q\left(\omega_{i}, \tau_{j}\right)=\frac{\omega_{i} U\left(\omega_{i}, \tau_{j}\right)}{P_{\mathrm{d}}\left(\tau_{j}\right)} \simeq \frac{16 \pi^{2} V}{\lambda_{j}^{3}} \frac{P_{\mathrm{R}}\left(\omega_{i}, \tau_{j}\right)}{P_{\mathrm{T}}\left(\omega_{i}, \tau_{j}\right)}
$$

where

$$
\begin{aligned}
\omega_{i} U\left(\omega_{i}, \tau_{j}\right) \propto & P_{\mathrm{T}}\left(\omega_{i}, \tau_{j}\right) V \\
& \times \sum_{\alpha}\left\langle\frac{\left|S_{21, \alpha}\left(\omega_{i} ; \tau_{j}\right)\right|^{2}}{1-\left|S_{22, \alpha}\left(\omega_{i} ; \tau_{j}\right)\right|^{2}}\right\rangle_{\Delta \omega} \\
P_{\mathrm{d}}\left(\omega_{i}, \tau_{j}\right) \propto & P_{\mathrm{T}}\left(\omega_{i}, \tau_{j}\right) R_{\mathrm{s}} S \\
& \times \sum_{\alpha}\left\langle 1-\left|S_{11, \alpha}\left(\omega_{i} ; \tau_{j}\right)\right|^{2}\right\rangle_{\Delta \omega}
\end{aligned}
$$

where $\alpha=x, y, z$, with $\langle\cdot\rangle_{\Delta f}$ denoting spectral averaging across $\left[\omega_{i}-\Delta \omega / 2, \omega_{i}+\Delta \omega / 2\right]$. Expressions (5) and (6) assume that the stored and dissipated energies are statistically homogeneous (because their estimation is based on local evaluation at two measurement locations for representing volumetric and surfacial aggregates), isotropic (because the antennas measure

\footnotetext{
${ }^{1}$ If ergodicity holds, then $Q$ in (1) equals $\langle Q(\tau)\rangle$ in a stirred cavity.
}

arbitrary 1-D projections of 3-D and 2-D rotationally invariant fields), and angularly delta correlated.

Following subsequent ensemble (i.e., stir) averaging with respect to $\tau_{j}$, the $m$ th moment $\left\langle Q^{m}\right\rangle$ is obtained, yielding the mean $\mu_{Q} \equiv\langle Q\rangle$ and variance $\sigma_{Q}^{2}=\left\langle Q^{2}\right\rangle-\langle Q\rangle^{2}$. Thus, the statistics of $Q$ are those of $Q(\tau)$ observed across $\left[f_{i}-\right.$ $\left.\Delta f / 2, f_{i}+\Delta f / 2\right]$. In principle, these can be calculated without spectral averaging, i.e., for $\Delta f=0$; however, in that case the spread tends to be larger. Therefore, $\Delta f / f_{i}$ should not be too small in order to make the estimation inaccurate. On the other hand, since $Q$ is defined for CW excitation and MT/MSRCs are strongly dispersive, $\Delta f / f_{i}$ should not be too large, in order for spectral averaging to be representative of ensemble stir averaging at $f_{i}$.

In our experiments, the S-parameters were measured at $N_{s}=1400$ uniformly spaced stir states (angles) $\tau_{j}$ spanning one full rotation. The two measured frequency ranges were from 0.1 to $6.1 \mathrm{GHz}$ and from 6 to $12 \mathrm{GHz}$, with a uniform step of $\delta f=150 \mathrm{kHz}$ at each $\tau_{j}$. The data for these 80,000 frequencies were subdivided into 80 blocks of 1000 frequencies for ease of processing. Within each block, spectral averaging was performed across $n=1$ to 480 contiguous frequency points, thus covering bandwidths $\Delta f=(n-1) \delta f$ ranging from zero $(n=1)$ to $72 \mathrm{MHz}(n=480)$ that yield $80 \times\lfloor 1000 / n\rfloor$ calculated values for each statistic of $Q$.

\section{B. Estimation}

From the spectrally aggregated data across $\Delta f$, the mean $\mu_{Q}$, standard deviation $\sigma_{Q}$ and coefficient of variation $\nu_{Q} \triangleq$ $\sigma_{Q} / \mu_{Q}$ for averaging of the $N_{s}$ stir states are extracted using two different methods. In fully empirical estimation, these statistics of $Q$ are computed as sample statistics directly, i.e., as weighted sums of the data through (4)-(6). The $2.5 \%$ and $97.5 \%$ boundaries $\xi^{-}$and $\xi^{+}$of a $95 \%$ CI for $Q$ are obtained by interpolating the empirical $\mathrm{CDF}^{2}$

On the other hand, semi-analytical estimation is based on the theoretical F-S model for the ratio distribution of $Q$ [2, eq. (14)]

$$
f_{Q}(q)=\frac{\left(\frac{2 M-1}{3 M} \mu_{Q}\right)^{2 M}}{\mathrm{~B}(3 M, 2 M)} \frac{q^{3 M-1}}{\left(q+\frac{2 M-1}{3 M} \mu_{Q}\right)^{5 M}} .
$$

For spectral averaging, the value of $M$ can be estimated either by the moment method (MME) using $\langle Q\rangle$ and $\left\langle Q^{2}\right\rangle$ or from a best fitting F-S CDF to the empirical CDF by maximum likelihood estimation (MLE) [7]. From this F-S CDF, estimates of $\mu_{Q}, \sigma_{Q}$ and $\nu_{Q}$ are obtained from their expressions in terms of $M$ [2, eqs. (15)-(17)]. The fitted CDF can be further used to estimate the $2.5 \%$ and $97.5 \%$ percentiles $\xi^{ \pm}$of a $95 \% \mathrm{CI}$ for $Q$ from [2, eqs. (25)-(26)].

In inverse $\mathrm{UQ}, M$ may be considered as a hyperparameter of the distribution of $\left\langle P_{\mathrm{R}}(\tau)\right\rangle_{V}$ in (3) within a Bayesian framework for $f_{Q}(q)=f_{Q \mid M}(q \mid m) f_{M}(m)$ [9, eq. (7)]. In

\footnotetext{
${ }^{2}$ Theoretical and empirical CDFs for the present data, from which the CI boundaries for $95 \%$ and other confidence levels can be deduced, were presented in [7] and [11].
} 
[2], the degrees of freedom parameter $M$ was interpreted as the average number of contributing cavity modes for $\mathrm{CW}$ excitation $(n=1)$ upon ensemble averaging (stirring). If we denote this value momentarily as $M_{0}$, then the generic $M$ in the case of additional spectral aggregation across $\Delta f$ is $M_{n-1} \lesssim n \cdot M_{0}$. In the latter expression, strict equality is achieved only when the data at all $n-1$ frequencies with contiguous spacings $\delta f_{i}$ are statistically independent. In practice, a fortiori in the case of large ohmic losses, the band of an individual cavity mode may overlap with those in the adjacent $\delta f_{i-1}$ and $\delta f_{i+1}$, whence significant frequency correlation can occur [7, Fig. 7], [11, Fig. 4] that may affect the estimation of $M_{0}$ from $M_{n-1}$.

\section{RESUlts}

Results for several summary statistics and CIs as a function of $M$ and $f$ are presented below. The validation of the theoretical F-S distribution model (7) is most vividly demonstrated by the $M$-characteristics, while frequency characteristics have greater practical use. The probabilistic model implies that stored and dissipated energies exhibit $\chi^{2}$ PDFs individually, whose degrees of freedom are governed by the single parameter $M$. Selected plots of results for both estimation methods are presented in the main text, whilst additional results are given in the supplementary data files. For the walls, a conductivity $\sigma_{\mathrm{w}}=3.8 \times 10^{5} \mathrm{~S} / \mathrm{m}$ and permeability $\mu_{\mathrm{w}}=\mu_{0}$ were assumed, as estimated from the empirical $Q_{\text {eff }}(f)$ presented in Sec. IV-C. However, since $\nu_{Q}$ and the CIs are expressed relative to $\mu_{Q}$, this scaling of $\mu_{Q}$ does not fundamentally affect the UQ.

\section{A. M-Characteristics}

1) Mean, Standard Deviation, and Coefficient of Variation: The statistics $\mu_{Q}, \sigma_{Q}$, and $\nu_{Q}$ are estimated from the same data subsets as those for estimating $M$. Thus, $\mu_{Q}(M), \sigma_{Q}(M)$ and $\nu_{Q}(M)$ regress these statistics against $M$ with frequency as a hidden variable. Since the $M(f)$-characteristic has previously been found to show relatively large fluctuations [7, Fig. 4], the characteristics $\mu_{Q}(M), \sigma_{Q}(M)$ and $\nu_{Q}(M)$ may likewise be expected a priori to show considerable spread.

Figs. S12(a)-(d) in the supplementary data indicate that, for arbitrary spectral widths $n$, the values of the fully empirical $\mu_{Q}(M)$ and $\sigma_{Q}(M)$ (represented as dots) are considerably more widely spread than the semi-analytic estimates (circles). The values are primarily concentrated near the top of the diagram, a fortiori for larger spectral averaging $(n \gg 1)$ where both methods show close agreement. For $\sigma_{Q}(M)$, a pronounced decrease exists on average.

Despite the large spread of $\mu_{Q}(M)$ and $\sigma_{Q}(M)$, Fig. 1 shows that the characteristic of their ratio $\nu_{Q}(M)$ is strongly localized and exhibits a considerably smaller spread than that in Figs. S12(a)-(d). The empirical $\nu_{Q}(M)$ is in close agreement with the semi-analytic and theoretical $\nu_{Q}(M)$, the latter being asymptotically proportional to $1 / \sqrt{M}$ for an ideal F-S $f_{Q}(q)$ with $M \gg 1$ [2, eq. (20) and Fig. 2]. The close agreement of the fully empirical sample statistic $\nu_{Q}(M)$ with the theoretical $\nu_{Q}(M)$ confirms the consistency of the values of $M$ and $\nu_{Q}$ estimated from the data.

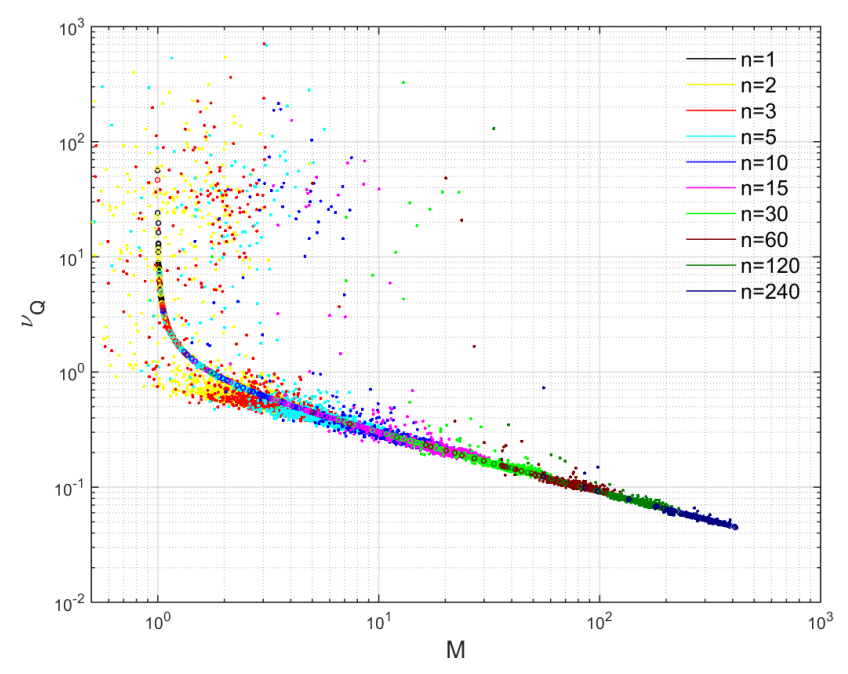

Fig. 1: Semi-analytic (circles) and fully empirical (dots) coefficient of variation $\nu_{Q}=\sigma_{Q} / \mu_{Q}$ as a function of $M$ from 0.1 to $12 \mathrm{GHz}$. See supplementary data for plots of $\mu_{Q}(M)$ and $\sigma_{Q}(M)$.

2) CI Interval: Fig. 2 shows estimated $\xi^{-}$and $\xi^{+}$for semianalytic and empirical $\eta \%$ CIs of $Q$ for $\eta=95$, normalized by $\langle Q\rangle$ and regressed on $M$. For large $M$, the figure demonstrates excellent agreement between both methods and also with the theoretical CIs [2, Fig. 3(b)]. For point values, i.e., without spectral averaging $(n=1)$, the semi-analytic normalized upper boundary $\xi_{0.975}^{+} /\langle Q\rangle$ reaches a maximum value of 4.997, which is commensurate with the theoretical maximum at $M \simeq 0.763$ shown in [2, Fig. 3(b)]. On the other hand, the fully empirical $\xi_{0.975}^{+} /\langle Q\rangle-$ not being constrained by the permissible values of $M$ - exhibits still larger values beyond 4.997 for $M<1$ when $n \leq 3$. For $n=2$ and low $M$, good agreement exists with theory as $\xi^{+}(M)$ and $\xi^{-}(M)$ tend to zero when $M$ approaches $1 / 2$ [2, Fig. 3(b)].

It is worth noting that the scatter of the "funnel" in Fig. 2 is lower for the fully empirical CI boundaries (dots). Thus, in this case, the limited accuracy of the estimated $M-$ rather than any inaccuracy that is attributable to the F-S model itself - is a major cause of the discrepancy with respect to the empirical sample CDF. Even a small amount of frequency averaging $(n>1)$ is seen to result in considerably sharper CI boundaries compared to the more fuzzy boundaries for $n=1$.

When $M$ decreases, the scatter of the boundaries around their theoretical characteristics in Fig. 2 increases for both semi-analytical and fully empirical estimates. This is expected on account of the then reduced spectral averaging (lower $n$ ), where low values of $M$ are more prominent. This effect of small sample size is a manifestation of the increasing sampling error of the random CI boundaries $\Xi^{ \pm}$. For a sufficiently large number of independent samples $(N \gg 1)$, the standard error 
of the boundaries of an $\eta \% \mathrm{CI}$ for $Q$ can be estimated from its asymptotic expression as [15, sec. 3.1.4], [16, sec. 28.5]

$$
s_{\Xi^{ \pm}}=\frac{\sqrt{1-(\eta / 100)^{2}}}{2 \sqrt{N} f_{Q}\left(\xi^{ \pm}\right)}
$$

where the values $\xi^{ \pm} \triangleq F_{Q}^{-1}((1 \pm(\eta / 100)) / 2)$ for a chosen confidence level $\eta \%$ are obtained numerically [2, eq. (25)]. Similarly, the standard error of the median $\Xi^{0} \triangleq F_{Q}^{-1}(1 / 2)$ with sample values $\xi^{0}$ follows from (8) with $\eta=0$ as $s_{\Xi^{0}}=$ $1 /\left[2 \sqrt{N} f_{Q}\left(\xi^{0}\right)\right]$. For the CI width $\Xi^{+}-\Xi^{-}$, the asymptotic standard error is

$$
\begin{aligned}
& s_{\Xi^{+}-\Xi^{-}}=\frac{1}{2 \sqrt{N}} \\
& \times \sqrt{\frac{1-(\eta / 100)^{2}}{\left[f_{Q}\left(\xi^{+}\right)\right]^{2}}-\frac{[1-(\eta / 100)]^{2}}{f_{Q}\left(\xi^{+}\right) f_{Q}\left(\xi^{-}\right)}+\frac{1-(\eta / 100)^{2}}{\left[f_{Q}\left(\xi^{-}\right)\right]^{2}}} .
\end{aligned}
$$

For the F-S distribution of $Q$, these standard errors are shown as a function of $M$ in Fig. 3(a), after renormalization by $\sqrt{N}$ and a further normalization by the ensemble mean $\langle Q\rangle$. The figure demonstrates the asymmetry between the standard errors for the upper vs. lower CI bound, owing to the skewness of $f_{Q}(q)$ itself. For the median, $s_{\Xi^{0}}$ is smaller than $s_{\Xi^{-}}$when $M>5.80$ and always smaller than $s_{\Xi^{+}}$irrespective of $M$. Fig. 3(b) shows corresponding CIs of $\Xi^{0}$ and $\Xi^{ \pm}$for coverage factors $k^{+}=k^{-}=k^{0}=0.25$ that are the same values as those in Fig. 2 and for $^{3} N=M / 1.5$ [7, Fig. 8], in the case where values of $Q$ are obtained by spectral sampling.

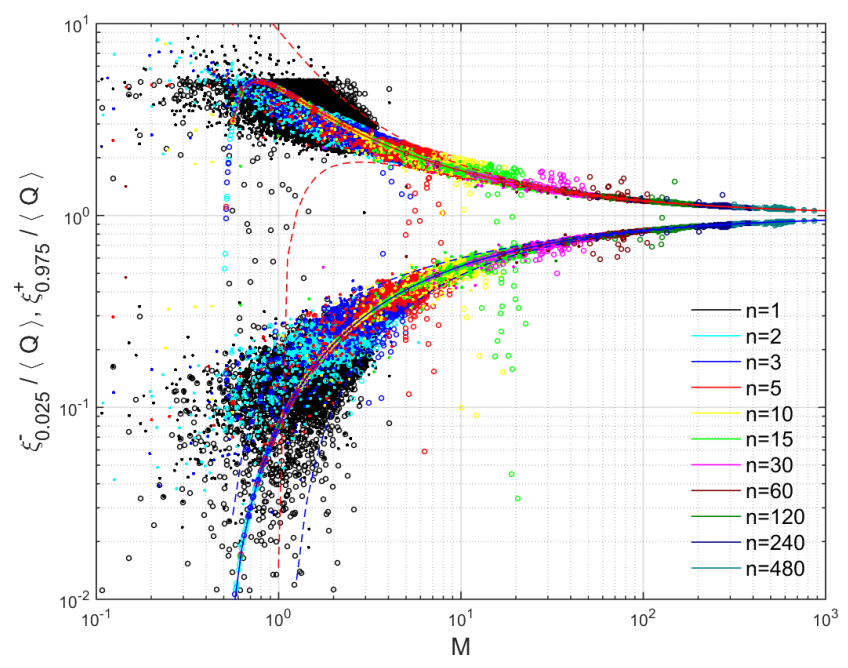

Fig. 2: Mean-normalized semi-analytic (circles) and fully empirical (dots) upper and lower boundaries for $95 \% \mathrm{CI}$ of $Q$ as a function of $M$ from 0.1 to $12 \mathrm{GHz}$. Dashed curves represent sample CIs for the upper $\left(\Xi^{+}\right.$; red) and lower $\left(\Xi^{-}\right.$; blue) ensemble CI boundaries, based on Fig. 3(b).

For fully empirical estimation, the CI boundaries for the

${ }^{3}$ Here $N=n$ is assumed, corresponding to $N$ independent estimates of $Q$ without spectral averaging $(n=1)$.

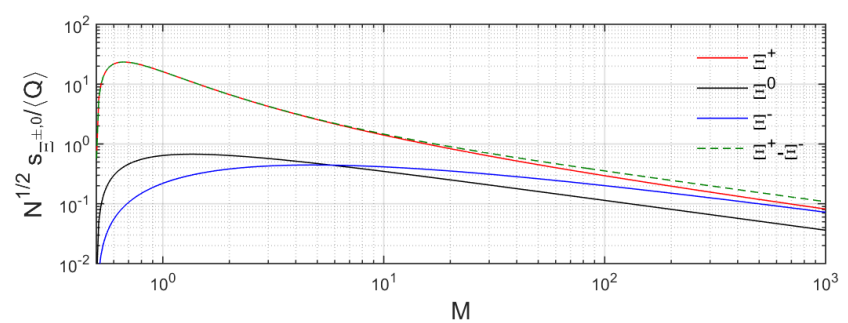

(a)

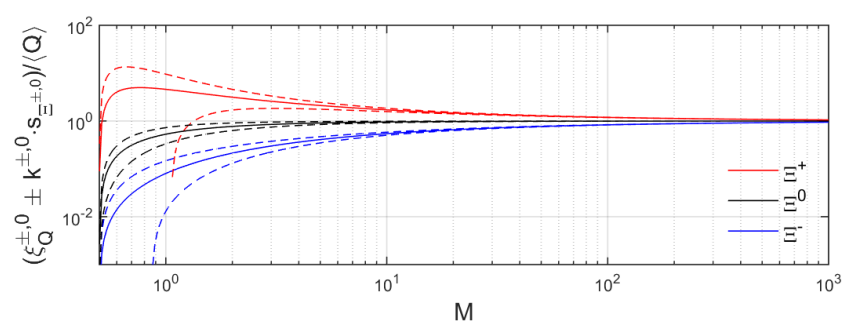

(b)

Fig. 3: (a) Mean-normalized theoretical asymptotic standard error $s$ for the median $\left(\Xi^{0}\right)$, upper $\left(\Xi^{+}\right)$and lower $\left(\Xi^{-}\right)$CI boundaries and for the width $\left(\Xi^{+}-\Xi^{-}\right)$of $95 \%$ CIs for F-S distributed $Q$ as a function of $M$, renormalized by $\sqrt{N}$ and valid for $N \gg 1$. (b) Meannormalized theoretical ensemble median, upper and lower boundaries of $95 \%$ CIs for F-S distributed $Q$ as a function of $M$ (solid curves), with boundaries of associated sample CIs based on $s_{\Xi \pm, 0}$ in figure (a) for $k^{+}=k^{-}=k^{0}=0.25$ (dashed curves in same colors).

ensemble F-S model as a function of $M$ become fuzzier for smaller values of $M$, as shown in Fig. 2. For CW excitation in the theoretical model [2], a small $M$ typically implies low frequency operation and/or small modal overlap. A sampling PDF for $Q$ or its approximation should then be used [14]. Whereas estimates of the semi-analytic CI boundaries for $n=5$ still conform to the ensemble F-S model, the empirical boundaries do no longer reproduce the skew in the theoretical characteristic (blue) when $n \leq 3$. Specifically, for $n=3$ and $M \sim 1 \ldots 3$, the empirical lower $2.5 \%$ boundary is higher than the theoretical one, while the upper boundary shows a bifurcation between semi-analytic values (being typically too large) and empirical values (being too small).

When normalizing $\xi^{ \pm}$by $Q_{\infty}$ instead of $\langle Q\rangle$ or removing normalization, the skewness of the CI is inverted (cf. Figs. S13 and S14 in the supplementary data files).

\section{B. Frequency Characteristics}

1) Distribution Parameter $M$ : Fig. 4(a) shows dispersion characteristics of $M(f)$ for selected $n$, based on the first two moments of the measured S-parameters (MME); recall that MLE of $M(f)$ yields very similar characteristics [7, Fig. 4]. The rapid fluctuations of $M(f)$ are a manifestation of the fluctuations of the contributing modes and of the modal spacing-to-bandwidth ratio in the overmoded regime. At high frequencies, where energy storage dominates dissipation, $M(f)$ for $n=1$ fluctuates around 2.5. The latter is the 
value expected for the $\chi_{6 N}^{2}$ distribution for storage when $N=n=1$ (CW excitation) for which $\nu_{\chi_{6 N}^{2}}=1 / \sqrt{3 N}$ (cf. [15, eq. (12)]), whence $M=(5 / 6) / \nu_{Q}^{2}=2.5$ for the F-S PDF $f_{Q}(q)$ (cf. [7, eq. (17)]). For $n \gg 1$, however, it is seen that $M(f \rightarrow+\infty) \simeq n<2.5 n$. This is a manifestation of the increased spectral correlation between the $n$ sample values within a band of the fixed width $\Delta f=(n-1) \delta f$ (cf. [7, Fig. $7]$ ). It remains to be investigated in future work to what extent these levels of spectral correlation agree with the pertinent spatial correlation for $U$ and $P_{\mathrm{d}}$ (second-order ergodicity). Note that in the low- to mid-frequency range, Bessel $K$ sampling distributions for $U$ and $P_{\mathrm{d}}$ [14] are expected produce a more accurate model.

The physical modelling of $M(f)$ is complicated by several factors. Cavity loss and leakage (through wall loss and antenna ports) causes deviations from the asymptotic Weyl law for the density of spectral lines in closed and open lossless cavities [18]. Increased modal overlap [19], [20] yields mode nonorthogonality, larger modal exchange energy, and ultimately the occurrence of resonance trapping and superradiance [21], [22]. In turn, this leads to increased spectral rigidity and spectral correlation between resonant modes compared to a closed cavity. This limits the increase of the effective number of independent modes as a measure for the number of degrees of freedom in the spectral aggregation method. In addition, dispersion in the coupling between the cavity field and the antennas affects the measurement of the absorption spectrum [23].

Whereas $M(f)$ in Fig. 4(a) is specifically associated with the F-S pdf (7), it is interesting to compare these values to the approximate number of degrees of freedom estimated from the Welch-Satterthwaite (W-S) formula [24] for the pooled spectral variances of the individual stir states, viz.,

$$
M_{\mathrm{W}-\mathrm{S}}\left(f_{i}\right)=\frac{\left(\sum_{j=1}^{N} \frac{s_{j}^{2}}{\nu_{j}+1}\right)^{2}}{N \sum_{j=1}^{N}\left[\left(\frac{s_{j}^{2}}{\nu_{j}+1}\right)^{2} / \nu_{j}\right]} .
$$

Here, $s_{j}^{2}\left(f_{i}\right)$ is the sample variance of $Q\left(f_{i}\right)$ at stir state $\tau_{j}$, for data taken across the $n$ frequencies within $\left[f_{i}-\right.$ $\left.\Delta f / 2, f_{i}+\Delta f / 2\right]$ and estimated based on (4). For simplicity, here we assume the fixed maximum values $\nu_{j}\left(f_{i}\right)=n-1$ and $N\left(f_{i}\right)=N_{s}$ for all $i$ and $j$, although these should be revised downward across frequency or stir state per frequency interval, respectively (particularly for small $n$ and low $f_{i}$ ), to account for correlation between stir states. Notwithstanding, the results in Fig. 4(b) confirm the rapid average increase of $M_{Q}(f) \equiv M_{\mathrm{W}-\mathrm{S}}(f)$ until approximately $1 \mathrm{GHz}$ and its approach $M(f \rightarrow+\infty) \sim n$ for $n \gg 1$, as expected.

2) Mean, Standard Deviation, and Coefficient of Variation:

a) Local Spectral Averaging: The characteristics $\mu_{Q}(f)$, $\sigma_{Q}(f)$ and $\nu_{Q}(f)$ are obtained directly from the $S_{k \ell}(f)$ : for their plots, there is now no intermediary via $M$.

Fig. 5(a) shows that the semi-analytic $\mu_{Q}(f)$ depends only weakly on $n$, as manifested by the $\mu_{Q}(f \mid n)$ for different $n$ be-

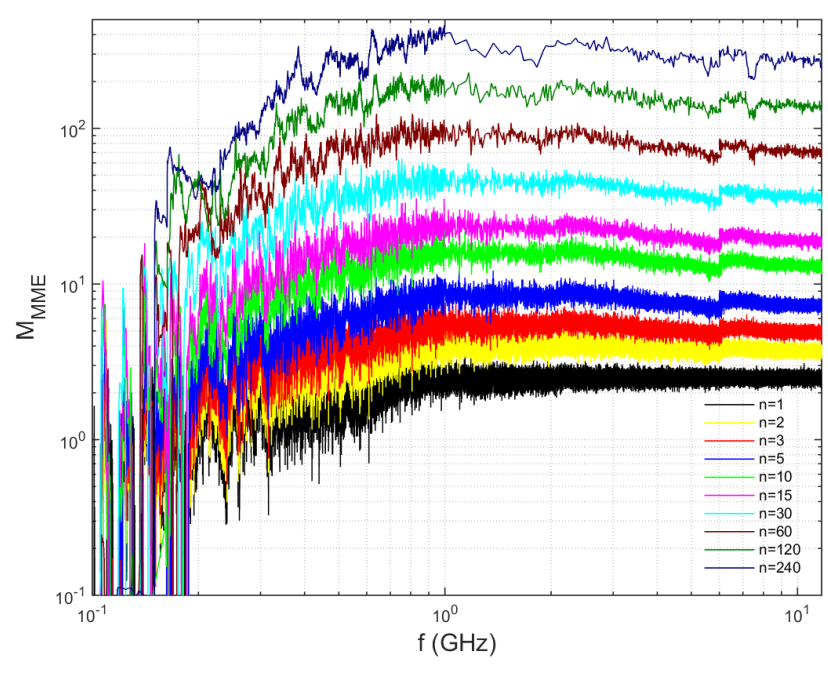

(a)

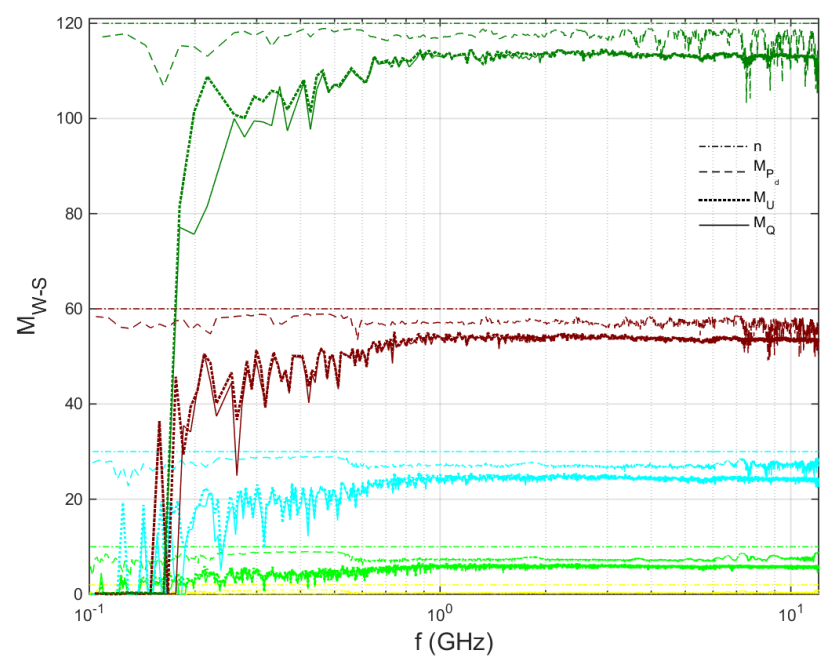

(b)

Fig. 4: (a) Moment method based estimates of empirical $M(f)$ for $Q$ at selected values of $n$. Above $1 \mathrm{GHz}$, the characteristics are shown with a 1:n reduction in spectral resolution (adjacent frequency intervals). Discontinuities at $6 \mathrm{GHz}$ are a result of displacement of the receiving antenna. (b) Welch-Satterthwaite estimates of empirical $M(f)$ for $Q, U$ and $P_{d}$ at $n=2,10,30,60$ and 120 (same color scheme), shown with 1:n resolution reduction.

ing tightly grouped. For the empirical $\mu_{Q}(f)$, the dependence on $n$ is still weaker, with fluctuations largely overlapping. On this basis, there is considerable freedom in selecting $n$. At low frequencies, the antenna's conduction losses, impedance mismatch, and reactive energy stored in its near field contribute significantly to the overall $Q$, in particular in the untuned (i.e., neither resonant nor antiresonant) regimes of the antenna [23, Sec. IV-C].

Figs. S15(c)-(d) in the supplementary data files show that $\sigma_{Q}$ exhibits a qualitatively similar frequency dependence to 
$\mu_{Q}$, particularly at high frequencies. Good numerical correspondence between semi-analytic and empirical $\sigma_{Q}(f)$ is achieved above $10 \mathrm{GHz}$, a fortiori when $n$ is large. This demonstrates the beneficial effect that the increase of the spectral averaging has on lowering the sampling error at low frequencies. The semi-analytic $\sigma_{Q}(f)$ exhibits a reduced spread with increasing $n$.

Fig. 5(b) shows that $\nu_{Q}(f)=\sigma_{Q}(f) / \mu_{Q}(f)$ exhibits even better agreement between semi-analytical and empirical estimates than those for $\mu_{Q}(f)$ and $\sigma_{Q}(f)$ individually, notably also at lower frequencies where the discrepancies for $\mu_{Q}(f)$ are largely compensated by $\sigma_{Q}(f)$. Interestingly, $\nu_{Q}(f)$ exhibits a slight but distinct increase with frequency, a fortiori for larger $n$. This suggests that the values and dispersion of $\sigma_{Q}(f)$ increase more rapidly than for $\mu_{Q}(f)$. For ease of comparison, the semi-analytic and fully empirical $\mu_{Q}(f)$, $\sigma_{Q}(f)$ and $\nu_{Q}(f)$ are shown separately in Fig. S15 of the supplementary data.

b) Inverse Spectral Averaging: Fig. 6(a) shows the rescaled standard deviation

$$
\sigma_{Q}^{*}(f \mid n) \triangleq \sqrt{n} \sigma_{Q}(f)
$$

i.e., in which $\sigma_{Q}(f)$ is compensated for its reduction as a result of sample size, caused by spectral aggregation with associated standard error $s_{\bar{Q}}=\sigma_{Q} / \sqrt{n}$. At high frequencies, the empirical $\sigma_{Q}^{*}(f \mid n)$ are seen to merge with the semianalytic characteristics, even though the former increase somewhat more rapidly than the latter because the data at the $n$ frequencies are not fully independent. This occurs for all $n$, a fortiori when $f$ and $n$ increase. A similar merger is found for $\nu_{Q}^{*}(f \mid n) \triangleq \sqrt{n} \nu_{Q}(f)$, shown in Fig. 6(b), whose spread across different $n$ is considerably smaller than for $\nu_{Q}(f)$ in Fig. 5(b). The residual spread in $\nu_{Q}^{*}(f \mid n)$ is mainly due to the spread of $\mu_{Q}(f)$ across $n$ as witnessed from Fig. 5(a).

Importantly, the close correspondence demonstrates that the effect of spectral averaging on the UQ of $Q$ is largely ${ }^{4}$ invertible, enabling retrieval of the "fundamental" $\sigma_{Q}(f)$ for $n=1$ (point values). Such inverse averaging [17] is meaningful because $\sigma_{Q}(f)$ for $n=1$ cannot be directly obtained as no spectral averaging then applies. From $\nu_{Q}^{*}(f \mid n)$ in Fig. 6(b) taken at $n=1$, inverse spectral averaging shows that

$$
\nu_{Q}(f) \simeq 0.65 \ldots 0.8
$$

as lower values between 1 and $12 \mathrm{GHz}$, while this ratio is considerably larger (by up to one order of magnitude, approximately) below $1 \mathrm{GHz}$. The residual high-frequency dependence of $\nu_{Q}^{*}(f)$ for different $n$ is a result of the remaining dependence of $\mu_{Q}(f)$ on $n$, rather than being an artefact of antenna $Q$ or impedance mismatch, because the ratio would compensate for the effect of any such cause. Note further that, after an expected sharp decrease of $\nu_{Q}^{*}(f)$ below $1 \mathrm{GHz}$ where the model is not expected to be highly accurate, $\nu_{Q}^{*}(f)$

\footnotetext{
${ }^{4}$ The inversion is not perfect because of the remaining weak dependence of $\mu_{Q}(f)$ on $n$, which is not compensated for in $\sqrt{n} \nu_{Q}(f)$.
}

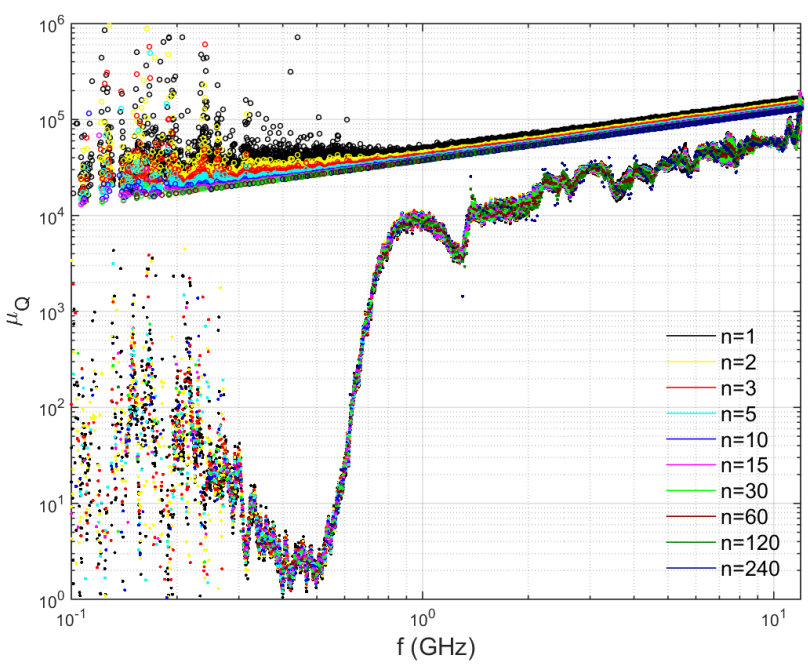

(a)

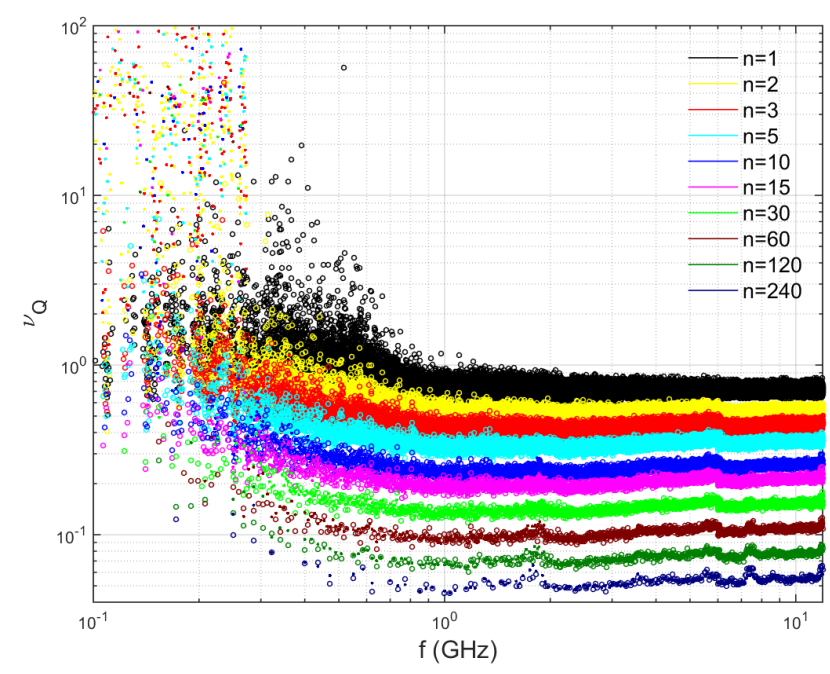

(b)

Fig. 5: Semi-analytic (circles) and fully empirical (dots) statistics of $Q$ as a function of $f$ from 0.1 to $12 \mathrm{GHz}$ for $\sigma_{\mathrm{w}}=3.8 \times 10^{5}$ S/m: (a) mean $\mu_{Q}(f)$, (b) coefficient of variation $\nu_{Q}(f)$.

does not approach zero because the value of $M$ saturates with increasing frequency.

In summary, the empirical frequency characteristics $\sigma_{Q}^{*}(f)$ and $\nu_{Q}^{*}(f)$ in Fig. 6 indicate that, regardless of the F-S model for $f_{Q}(q)$ and associated estimates of $M$ :

- the absolute and relative uncertainties of $Q$ scale approximately as $1 / \sqrt{n}$;

- the point values of the absolute uncertainty of $Q$ (i.e., for $n=1$ ) can be deduced consistently by inverse spectral averaging (removal of frequency averaging);

- the relative uncertainty of $Q$ slowly increases with frequency, in our experimental results above $1 \mathrm{GHz}$.

3) CI Interval: Fig. 7 shows that, in comparison with their dependence on $M$, the boundaries $\xi^{ \pm}(f)$ of the semi- 


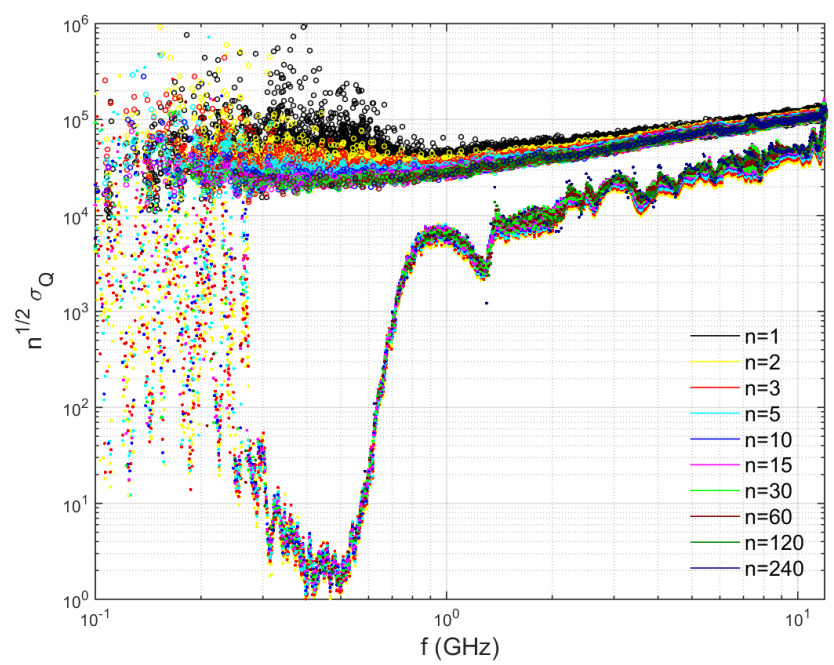

(a)

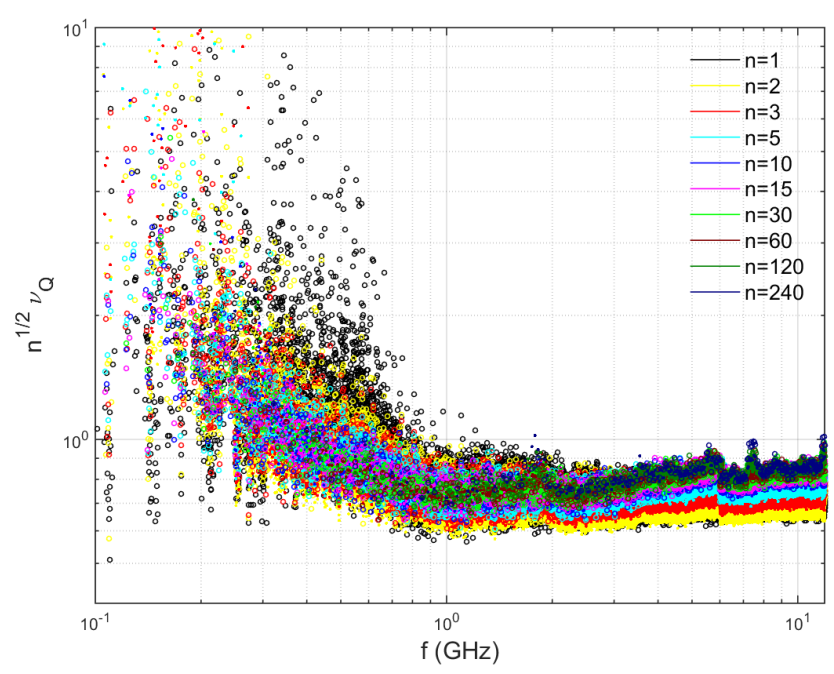

(b)

Fig. 6: Renormalized statistics of $Q$ as a function of $f$ from 0.1 to $12 \mathrm{GHz}:$ (a) $\sqrt{n} \sigma_{Q}$, (b) $\sqrt{n} \nu_{Q}$.

analytic $95 \% \mathrm{CI}$ are more irregularly spread. Compared to the fully empirical CI, the upper boundary of the semianalytic $\mathrm{CI}$ is somewhat higher as well as fuzzier, whereas the lower boundary is also higher but more localized. While the empirical $\mu_{Q}(f)$ spreads markedly when frequency decreases below $700 \mathrm{MHz}$, the CI remains accurate down to about 200 $\mathrm{MHz}$. This indicates that high overmoding is not a prerequisite for enabling accurate UQ of $Q$.

Unlike $\sigma_{Q}(f)$ and $\nu_{Q}(f)$, the CI boundaries for $n>1$ cannot be easily matched to those without spectral averaging $(n=1)$ via renormalization. The reason is the pronounced negative skewness of $f_{Q}(q)$, causing these boundaries not to scale symmetrically in accordance with $1 / \sqrt{n}$.

Similar to the previously mentioned small increase of
$\nu_{Q}(f)$, the results suggest that the CI shows a marginal but persistent widening with increasing frequency. Further investigation should establish whether this increase is systematic beyond the measurement uncertainty, and whether it is a property of the chamber $Q$, an antenna effect, or otherwise.

Plots for the mean-normalized boundaries for the CI are shown in Fig. S18 in the supplementary data files. Further

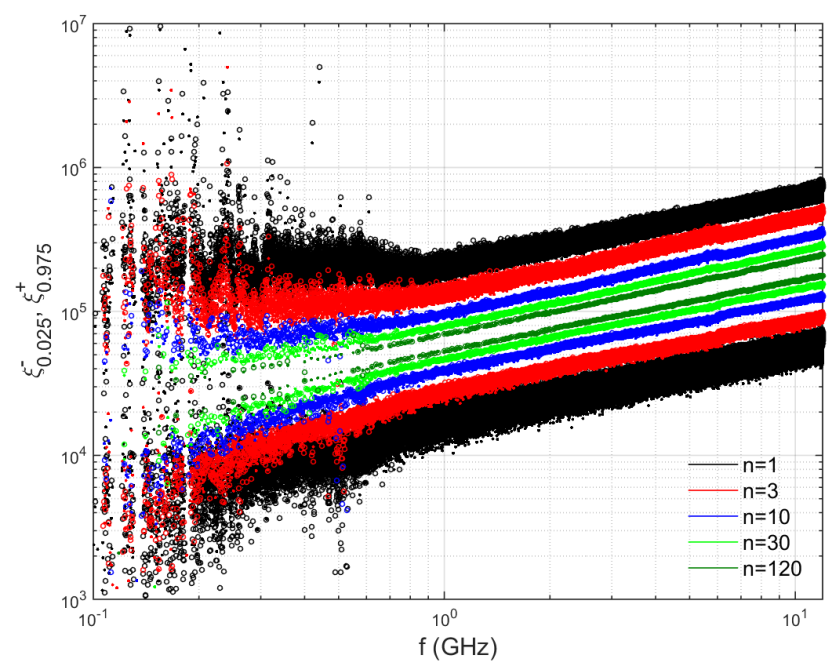

Fig. 7: Non-normalized semi-analytic (circles) and fully empirical (dots) upper and lower boundaries of $95 \% \mathrm{CI}$ of $Q$ as a function of $f$ from 0.1 to $12 \mathrm{GHz}$.

data analysis has indicated that the estimate of $\mu_{Q}$ is rather insensitive to the precise number of frequencies $n$ in the spectral averaging procedure.

For nonnegligible averaging bandwidths $(\Delta f / f \nless 1)$, the dispersion of MT/MSRCs in (3) causes spectral averaging to be not approximately uniform, but weighted according to $\sqrt{f}$ for $Q$ (or, equivalently, $f^{-5 / 2}$ for the insertion loss). This leads to inaccurate estimates of $\mu_{Q}(f)$ and $\sigma_{Q}(f)$. Because of this deviation from a $\sqrt{n}$-scaling law, it is recommended to apply spectral averaging using relatively small $n(n<10)$ in order to estimate the renormalized $\sigma_{Q}^{*}$ for $n=1$.

\section{C. $Q_{\mathrm{eff}}$ and $Q_{\infty}$ vs. Theoretical and Experimental Mean $Q$}

The effective (or composite) quality factor, $Q_{\text {eff }}=$ $\langle\omega U\rangle /\left\langle P_{\mathrm{d}}\right\rangle$ [4], is in general different from the statistical mean $\langle Q\rangle$, as $Q_{\text {eff }}$ exhibits a negative bias with reference to $\langle Q\rangle$ that increases with decreasing $M$ or $f$ [2]. On the other hand, $Q_{\text {eff }}\left(f_{i}\right)$ uses stir averaged values $\left\langle\left|S_{k \ell}\left(f_{i}\right)\right|^{2}\right\rangle$, thus only requiring a scalar analyzer (or alternatively a power meter measuring $P_{\mathrm{R}}$ and $P_{\mathrm{T}}$ ) with averaging. This obviates the need for evaluating and storing each $\left|S_{k \ell}\left(f_{i}, \tau_{j}\right)\right|^{2}$ at each $\tau_{j}$. Furthermore, provided the ensemble averaged wave impedance inside the MT/MSRC equals the free-space value $\eta_{0}$, the average $\left\langle\left|S_{11}\left(f_{i}\right)\right|^{2}\right\rangle$ (or $\left\langle P_{\mathrm{R}}\right\rangle$ ) can be approximated by its value for a single measurement in an anechoic chamber, thereby neglecting the interaction between the antennas and 
the MT/MSRC. These features make $Q_{\text {eff }}$ a popular alternative for $\langle Q\rangle$, even though it is less amenable to statistical analysis and UQ of $Q$. As a ratio of averages of $\omega U$ and $P_{\mathrm{d}}$, the fluctuation of $Q_{\text {eff }}$ is governed by their standard errors $s / \sqrt{N}$.

Fig. 8 compares various theoretical and experimental estimates of the $\langle Q\rangle$ and $Q_{\text {eff }}$. Here, $Q_{\text {eff }}(f)$ employs $\left\langle\left|S_{k k}\right|^{2}\right\rangle$ [15, sec. 4.1.2.1], while $Q_{2 \text { ant }}(f)$ uses $\left|\left\langle S_{k k}\right\rangle\right|^{2}$ instead, which is representative of free-space conditions [6]. The figure indicates that this difference in averaging method produces only a small bias that is weakly dependent on frequency. It is further observed that even for well-matched horn antennas, the theoretical $\sqrt{f}$-dependence of $Q(f)$ is approached fairly slowly, from $7 \mathrm{GHz}$ upwards. Potential sources of the spectral discrepancy are the typical decrease of $\mu_{\mathrm{w}}(f)$, the dependence of $\mu_{\mathrm{w}}$ on machining and forming processes during steel manufacturing, and the dependence of $\mu_{\mathrm{w}}$ on field strength (nonlinearity). Surface roughness, which yields a somewhat larger dependence of losses on frequency than that from $\delta_{\mathrm{w}}(f)$, may produce a secondary effect at millimetre wavelengths. These additional sources of loss and fluctuations also yield larger uncertainty of $Q$ at higher $f$.

When using a pair of mismatched short dipole array antennas [7, Fig. 2], $Q_{\text {eff }}(f)$ is close to $\mu_{Q, \text { emp }}(f)$, except again well below $1 \mathrm{GHz}$ where $M$ is small [2, Fig. 2 and eq. (24)], [7, Fig. 4]. By comparison, when using dual-ridge horn antennas instead, $Q_{\text {eff }}(f)$ exhibits a smaller ripple at higher frequencies than for the dipoles, as well as a ripple comparable to that for the theoretical F-S $\mu_{Q}(f)$.

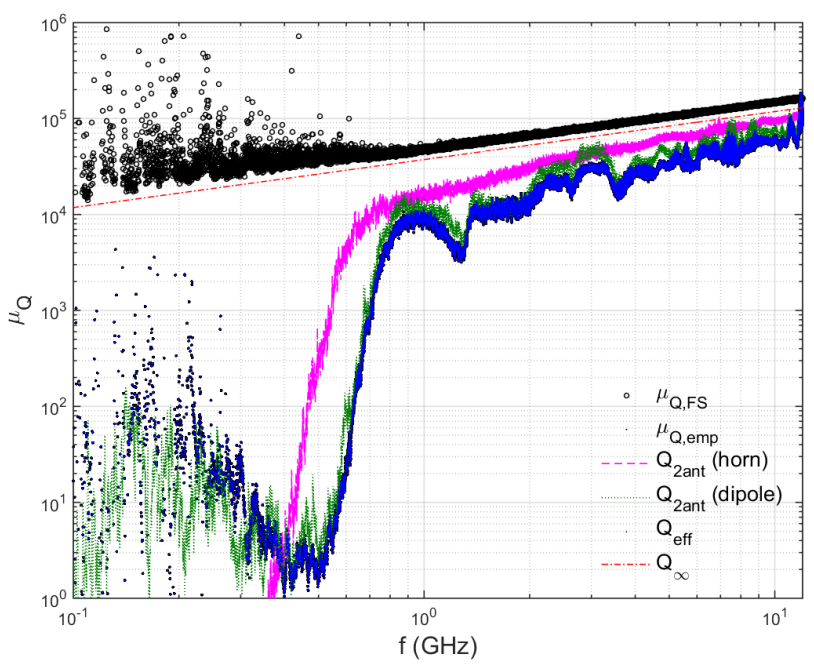

Fig. 8: Estimated theoretical and measured values of average measures of $Q$ : statistical mean $\mu_{Q}$, effective $Q_{\text {eff }}$ and asymptotic $Q_{\infty}$ for $\sigma_{\mathrm{w}}=3.8 \times 10^{5} \mathrm{~S} / \mathrm{m}$ without spectral averaging $(n=1)$.

Fig. 9 shows the relative difference of theoretical estimates for the mean $Q$, expressed as

$$
\varepsilon_{\text {theo }}(f) \triangleq \frac{\left|Q_{\infty}(f)-\mu_{Q, \mathrm{FS}}(f)\right|}{\mu_{Q, \mathrm{FS}}(f)}
$$

where $\mu_{Q, \mathrm{FS}}$ is the semi-analytic F-S mean $\langle Q\rangle$. The figure confirms that the merger of theoretical values saturates at a level that depends on $n$, e.g., $\varepsilon_{\text {theo }} \simeq 20 \%$ for $n=1$ while $\varepsilon_{\text {theo }}<1 \%$ for $n \geq 40$. Saturation occurs because $\left(\mu_{Q, \mathrm{FS}}-Q_{\infty}\right) / \mu_{Q, \mathrm{emp}}=1 /(2 M)$ [2, Fig. 2] and because of the saturation of $M(f \rightarrow+\infty)$ itself, as previously observed in [7, Fig. 4]. Significantly, there is no discernible improvement of $\varepsilon_{\text {theo }}(f)$ above $1 \mathrm{GHz}$.

In addition, Fig. 9 compares empirical estimates through the normalized difference

$$
\varepsilon_{\mathrm{emp}}(f) \triangleq \frac{\left|Q_{\mathrm{eff}}(f)-\mu_{Q, \mathrm{emp}}(f)\right|}{\mu_{Q, \mathrm{emp}}(f)}
$$

where $\mu_{Q \text {,emp }}$ symbolizes the fully empirical mean $Q$. For $n=1$, the characteristics $\mu_{Q, \mathrm{emp}}(f)$ and $Q_{\mathrm{eff}}(f)$ overlap. For $n>1$, a discrepancy between $\mu_{Q \text {,emp }}(f)$ and $Q_{\text {eff }}(f)$ arises because the former employs $\left\langle\left|S_{k k}\right|^{2}\right\rangle$, while the latter uses $\left|\left\langle S_{k k}\right\rangle\right|^{2}$. The relative difference $\varepsilon_{\text {emp }}(f)$ is typically less than $1 \%$ from $2 \mathrm{GHz}$ upward for any $n$, while being of the order of $10 \%$ downward to about $300 \mathrm{MHz}$.

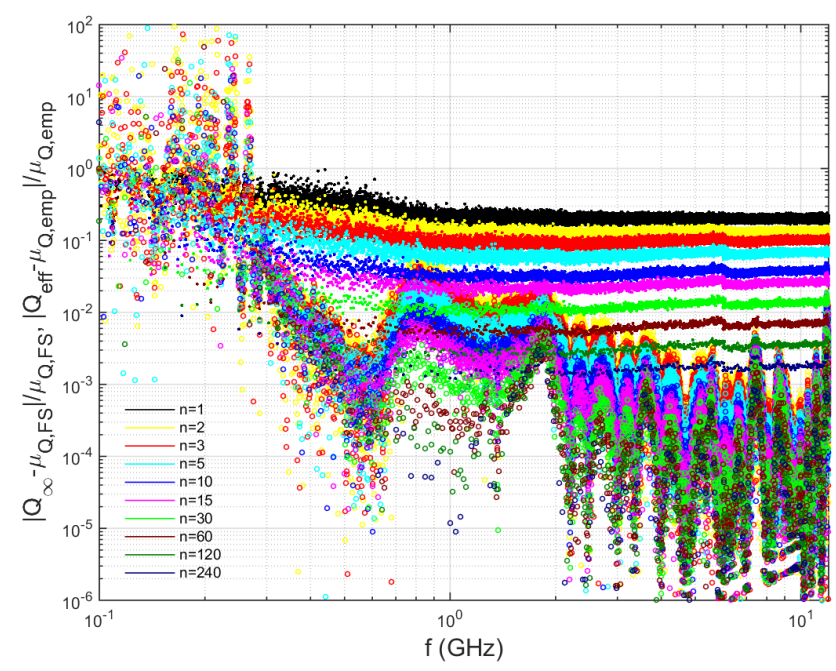

Fig. 9: (i) (dots) Relative difference $\varepsilon_{\text {theo }}$ between theoretical effective (asymptotic) $Q_{\infty}(f)$ and reference semi-analytic F-S average $\mu_{Q, \mathrm{FS}}(f)$, for selected values of the spectral averaging width $n=1+(\Delta f / \delta f)$. (ii) (circles) Relative difference $\varepsilon_{\text {emp }}$ between empirical effective (composite) $Q_{\text {eff }}(f)$ and reference empirical average $\mu_{Q \text {,emp }}(f)$, for selected values of $n$.

\section{Numerical Full-Wave Simulation}

For comparison, a static (unstirred) parallelepiped cavity with the same dimensions, wall conductivity $\left(3.8 \times 10^{5} \mathrm{~S} / \mathrm{m}\right)$ and permeability $\left(\mu_{\mathrm{w}}=\mu_{0}\right)$ as in the experiment was simulated using the finite-integration time-domain (FITD) method across a narrow band of 61 frequencies around $1 \mathrm{GHz}$. This scenario represents a spatial-spectral doubly-stochastic process $\left(\mathcal{P}_{R}, \mathcal{P}_{F}\right)$ without stirring. The interior magnetic field $\underline{H}(\underline{r}, f)$ and tangential field $\underline{H}_{\mathrm{t}}\left(\underline{r}_{S}, f\right)$ on the wall boundary were computed on a uniform mesh of $32,214(59 \times 26 \times 21)$ grid 
points, extracted from 4,264, 818 mesh cells. For these data, $Q(f)$ is obtained from [2, eq. (6)]

$$
Q(f)=\frac{2}{\mu_{\mathrm{w}, r} \delta_{\mathrm{w}}} \frac{\iiint_{V}|\underline{H}(\underline{r}, f)|^{2} \mathrm{~d} V}{\iint_{S}\left|\underline{H}_{\mathrm{t}}\left(\underline{r}_{S}, f\right)\right|^{2} \mathrm{~d} S}
$$

when the integrations are approximated by discrete sums over the mesh nodes. A Gaussian pulse excitation followed by inverse Fourier transformation of the time-domain data truncated at $-50 \mathrm{dBc}$.

Fig. 10 compares this $Q(f)$ for the simulated cavity with the previous estimated $\mu_{Q, F S}(f)$ of the stirred cavity for the measured S-parameter data of the local field based on the MME estimated empirical $M(f)$ at select $n$. The results confirm that increasing spectral aggregation result in a reduced bias of the estimated $Q(f)$, but at the expense of a reduced capability of finding the level and rate of the spectral fluctuations for the unstirred $Q(f)$. Furthermore, the figure confirms that a considerable reduction of the level of spectral fluctuations of the average $Q$ across frequency is achieved when mode stirring is applied, even in the absence of spectral aggregation $(n=1)$.

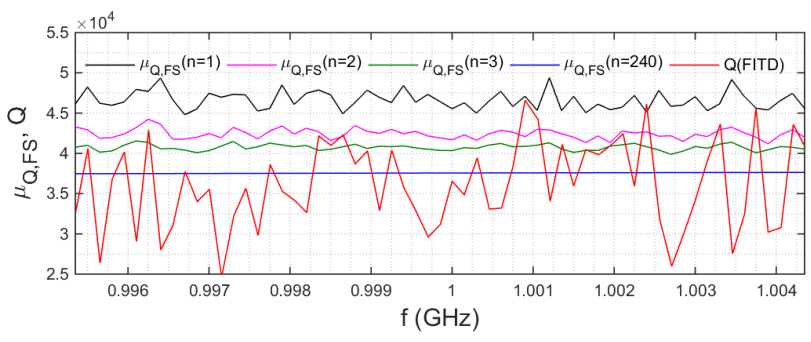

Fig. 10: $Q(f)$ for simulated unstirred cavity (red) vs. $\mu_{Q, \mathrm{FS}}(f)$ for measured stirred cavity at selected spectral aggregation lengths $n$.

\section{CIS FOR MAXimum AND Minimum $Q$}

Randomness and uncertainty of $Q$ are not only inherent to the stir process itself. They may also arise from differences in estimates of $Q$ obtained from multiple measurements at additional locations, in order to enlarge the data set with a view to increase the estimation accuracy [6, sec. III.C].

Strictly, the sampling PDF of $Q$ must be employed in the rigorous propagation of uncertainty when estimating statistics of $Q$-dependent field quantities. A simpler but approximate evaluation of their UQ can be performed by using deterministic values associated with maximum or minimum values of $Q$ instead, e.g., the maximum maximorum or maximum minimorum of $Q$ for $m$ receiver locations and $N$ independent stir states, in order to provide an indication of the dependence of the extremum of test field on the extreme values of $Q$. In EMC measurements and testing, the statistics of the maximum $Q_{\max }$ from $N$ independent samples of $Q$ are relevant in maintaining exposure levels of the field inside a MT/MSRC, whereas the minimum $Q_{\min }$ is relevant in the design of a MT/MSRC experiment and the amplification requirements for achieving a required minimum strength of the test field.
The CDFs of $Q_{\max }$ and $Q_{\min }$ are

$$
\begin{aligned}
F_{Q_{\max }}\left(q_{\max }\right) & =\left[F_{Q}\left(q_{\max }\right)\right]^{N}, \\
F_{Q_{\min }}\left(q_{\min }\right) & =\left[1-F_{Q}\left(q_{\min }\right)\right]^{N}
\end{aligned}
$$

respectively, where the F-S CDF of $Q$ [2] is $F_{Q}(q)=$ $1-I_{\gamma(q)}(2 M, 3 M)$ with $I_{\gamma(q)}$ representing a regularized incomplete beta function and

$$
\gamma(q) \triangleq\left(1+\frac{3 M}{2 M-1} \frac{q}{\langle Q\rangle}\right)^{-1}
$$

Differentiation of (16) and (17) yields their PDFs as

$$
\begin{aligned}
f_{Q_{\max }}\left(q_{\max }\right)= & N\left[1-I_{\gamma\left(q_{\max }\right)}(2 M, 3 M)\right]^{N-1} \\
& \times \frac{\left(\frac{2 M-1}{3 M}\langle Q\rangle\right)^{2 M}}{\mathrm{~B}(3 M, 2 M)} \frac{q_{\max }^{3 M-1}}{\left(q_{\max }+\frac{2 M-1}{3 M}\langle Q\rangle\right)^{5 M}} \\
f_{Q_{\min }}\left(q_{\min }\right)= & N\left[I_{\gamma\left(q_{\min }\right)}(2 M, 3 M)\right]^{N-1} \\
& \times \frac{\left(\frac{2 M-1}{3 M}\langle Q\rangle\right)^{2 M}}{\mathrm{~B}(3 M, 2 M)} \frac{q_{\min }^{3 M-1}}{\left(q_{\min }+\frac{2 M-1}{3 M}\langle Q\rangle\right)^{5 M}}
\end{aligned}
$$

Moment based statistics such as $\left\langle Q_{\max }\right\rangle$ and $\sigma_{Q_{\max }}$ follow by quadrature. Computationally, however, large powers of the beta functions of large orders rapidly lead to numerical inaccuracy. On the other hand, integration is not required when determining the (robust) median of $Q$ and CIs of $Q_{\max }$, which follow with (16) from those for $Q$ as

$$
\xi_{Q}^{ \pm}=F_{Q}^{-1}\left(\frac{1 \pm\left(\eta_{Q} / 100\right)}{2}\right)
$$

The CI boundaries for $Q_{\max }$ with $\eta_{Q_{\max }}=\eta_{Q} \triangleq \eta$ follow by a simple scaling of the $Q$-grades $\left[1 \pm\left(\eta_{Q} / 100\right)\right] / 2$ to their $N$ th root, i.e.,

$$
\xi_{Q_{\max }}^{ \pm}=F_{Q}^{-1}\left(\left(\frac{1 \pm\left(\eta_{Q_{\max }} / 100\right)}{2}\right)^{1 / N}\right) .
$$

Similarly, for $\eta_{Q_{\min }}=\eta_{Q}$, the boundaries for $Q_{\min }$ follow as

$$
\xi_{Q_{\min }}^{ \pm}=F_{Q}^{-1}\left(1-\left(\frac{1 \pm\left(\eta_{Q_{\min }} / 100\right)}{2}\right)^{1 / N}\right) .
$$

Fig. 11 shows $\xi_{Q_{\max }}^{ \pm}(N)$ for $\eta=95$ and 99 for the F$\mathrm{S}$ model. Both upper and lower boundaries increase with $N$, while the lower boundary rapidly approaches the upper one, i.e., combined narrowing and upward shifting. Conversely, decreasing tendencies hold for $\xi_{Q_{\min }}^{ \pm}(N)$ (not shown).

\section{CONCLUSION}

In this paper, it was demonstrated that, subject to limited spectral averaging and sufficiently large overmoding (as governed by a sufficiently large average number of excited cavity modes), the uncertainty quantification for the $Q$ of a MT/MSRC based on its theoretical Fisher-Snedecor probability distribution is consistent with the fully empirical (modelfree) estimates obtained from measured S-parameter sample 


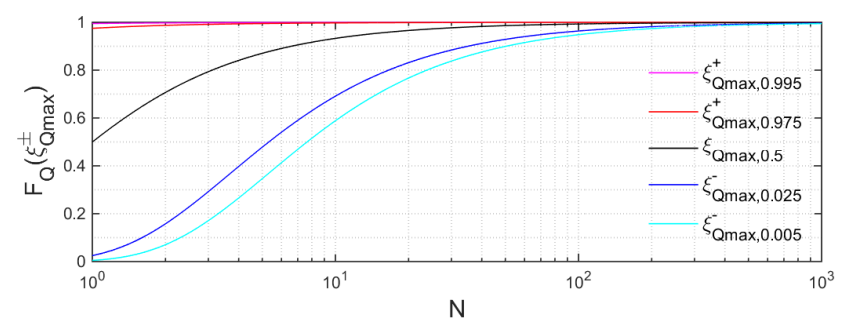

Fig. 11: $Q$-probabilities associated with the median, upper and lower boundaries $\xi$ of $95 \%$ and $99 \%$ CIs for $Q_{\max }$ as a function of number of independent samples $N$ for F-S distributed $Q$.

data [7]. As a function of frequency, the F-S and modelfree estimates were found to yield very similar values and characteristics for the coefficient of variation $\nu_{Q}(f)$ and for the boundaries of a $95 \%$ confidence interval $\xi^{ \pm}(f)$ of $Q$. As a function of the F-S distribution parameter $M$, the fully empirical results also confirm the theoretical characteristics derived in [2]. The distribution parameter $M$ was estimated empirically from the data, in order to maximize estimation accuracy because of the rapid spectral fluctuations of $M(f)$.

A numerical comparison between $\mu_{Q}(f)$ of the stirred cavity around $1 \mathrm{GHz}$ (estimated from the measured local fields) and the unstirred $Q(f)$ for a static cavity (based on the full-wave simulated magnetic field across the cavity) showed close agreement, even though the hybrid doubly-stochastic processes defined by both scenarios are physically different.

A physical-theoretical model for a priori estimating $M(f)$ is under investigation. The saturation or decrease of $M(f)$ with increasing frequency in Fig. 4 indicates that the Weyl law for a closed lossless cavity is incomplete to explain by itself the observed frequency dependence for our measurement configuration. The energy exchange for overlapping modes gives rise to spectral correlation that reduces the effective number of independent quasimodes governing $M$. The identification and precise counting of these modes by inversion of measured spectra of $S_{21}(f)$ and $S_{11}(f)$ [18], [26] could be instrumental in developing and improving a physical model for $M(f)$. The use of more elaborate Bessel $K$ sampling distributions for $U$ and $P_{\mathrm{d}}[14]$ can be expected to yield further improvements in the UQ of $Q$ in the mid-frequency range.

\section{REFERENCES}

[1] L. R. Arnaut, "Statistics of the quality factor of a rectangular reverberation chamber," IEEE Trans. Electromagn. Compat., vol. 45, no. 1, pp. 61-76, Feb. 2003.

[2] L. R. Arnaut and G. Gradoni, "Probability distribution of the quality factor of a mode-stirred reverberation chamber," IEEE Trans. Electromagn. Compat., vol. 55, no. 1, pp. 35-44, Feb. 2013.

[3] P. Corona, G. Latmiral, and E. Paolini, "Performance and analysis of a reverberating enclosure with variable geometry," IEEE Trans. Electromagn. Compat., vol. 22, no. 1, pp. 2-5, Feb. 1980.

[4] B.-H. Liu, D. C. Chang, and M. T. Ma, "Eigenmodes and the composite quality factor of a reverberating chamber," NBS Techn. Note 1066, U.S National Bureau of Standards, Boulder, CO, 1983.
[5] International Electrotechnical Commission, Electromagnetic Compatibility (EMC). Part 4: Testing and Measurement Techniques: Section 21: Reverberation Chambers, IEC 61000-4-21, 2nd ed., Geneva, Switzerland, Jan. 2011.

[6] P. Besnier, C. Lemoine, and J. Sol, "Various estimations of composite Qfactor with antennas in reverberation chamber," Proc. 2015 EMC Europe Int. Conf. Electromagn. Compat., 16-22 Aug. 2015, Dresden, Germany.

[7] L. R. Arnaut, M. Andries, J. Sol, and P. Besnier, "Evaluation method for the probability distribution of the quality factor of mode-stirred reverberation chambers," IEEE Trans. Antennas Propag., vol. 62, no. 8, pp. 4199-4208, Aug. 2014.

[8] T. E. Lovett, F. Ponci, and A. Monti, "A polynomial chaos approach to measurement uncertainty," IEEE Trans. Instr. Meas, vol. 55, no. 3, pp. 729-736, Jun. 2006.

[9] Y. M. Marzouk, H. N. Najm, and L. A. Rahn, "Stochastic spectral methods for efficient Bayesian solution of inverse problems," J. Comp. Phys., vol. 224, no. 2, pp. 560-586, Jun. 2007.

[10] C. E. Porter and R. G. Thomas, "Fluctuations of nuclear reaction widths," Phys. Rev., vol. 104, no. 2, pp. 483-491, Oct. 1956.

[11] L. R. Arnaut, M. Andries, J. Sol, and P. Besnier, "Measured probability distribution of the quality factor of mode-stirred reverberation chambers," Proc. 2014 EMC Europe Int. Conf. Electromagn. Compat. Göteborg, Sweden, 1-4 Sep. 2014.

[12] J. D. Jackson, Classical Electrodynamics. Wiley: New York, 1975.

[13] A. Gifuni, G. Ferrara, M. Migliaccio, and A. Sorrentino, "Estimate of the probability density function of the quality factor of mode tuned, source stirred and mode stirred reverberation chambers," IEEE Trans. Electromagn. Compat., vol. 57, no. 5, pp. 926-936, Oct. 2015.

[14] L. R. Arnaut, and G. Gradoni, "Probability distributions of the quality factor of spacecraft structures modelled as undermoded reverberation chambers," Proc. ESA Workshop on EMC, Venice, Italy, May 2012.

[15] L. R. Arnaut, "Measurement uncertainty in reverberation chambers I. Sample statistics," NPL Rep., TQE 2, 2nd ed., pp. 1-136, National Physical Laboratory, Teddington, U.K., Dec. 2008.

[16] H. Cramér, Mathematical Methods of Statistics. Princeton: Princeton University Press, 1946.

[17] L. R. Arnaut, "Effect of local stir and spatial averaging on the measurement and testing in mode-tuned and mode-stirred reverberation chambers," IEEE Trans. Electromagn. Compat., vol. 43, no. 3, pp. 305325, Aug. 2001

[18] A. Potzuweit, T. Weich, S. Barkhofen, U. Kuhl, H.-J. Stöckmann, and M. Zworski, "Weyl asymptotics: from closed to open systems," Phys. Rev. E, vol. 86, no. 6, pp. 066205, Dec. 2012

[19] V. V. Sokolov and V. G. Zelevinsky, "On the statistical theory of overlapping resonances," Phys. Lett. B, vol. 202, no. 1, pp. 10-14, Feb. 1988.

[20] A. Cozza, "Probability distributions of local mode-density fluctuations in an electromagnetic cavity," IEEE Trans. Electromagn. Compat., vol. 54, no. 5, pp. 954-967, Oct. 2012

[21] E. Persson, T. Gorin, and I. Rotter, "Decay rates of resonance states at high level density," Phys. Rev. E, vol. 54, no. 4, pp. 3339-3351, Oct. 1996.

[22] E. Persson, I. Rotter, H.-J. Stöckmann, and M. Barth, "Observation of resonance trapping in an open microwave billiard," Phys. Rev. Lett., vol 85, no. 12 , pp. 2478-2481, Sep. 2000.

[23] A. D. Yaghjian and S. R. Best, "Impedance, bandwidth and $Q$ of antennas," IEEE Trans. Antennas Propag., vol. 53, no. 4, pp. 1298 1324, Apr. 2005

[24] R. Willink, "A generalization of the Welch-Satterthwaite formula for use with correlated uncertainty components," Metrologia, vol. 44, no. 5, pp. 340-349, Oct. 2007.

[25] M. I. Andries, and L. R. Arnaut, "Numerical study of statistical properties of stored and dissipated energy in reverberant cavities," Proc. 2nd URSI Atlantic Meeting (AT-RASC), paper S-EABK-5.

[26] F. Sarrazin and E. Richalot, "Cavity modes inside a mode-stirred reverberation chamber using the matrix pencil method," Proc. 11th Eur. Conf. Antennas Propag. (EuCAP), Paris, 2017, pp. 620-622. 
SUPPLEMENTARY DATA I: $M$-CHARACTERISTICS 


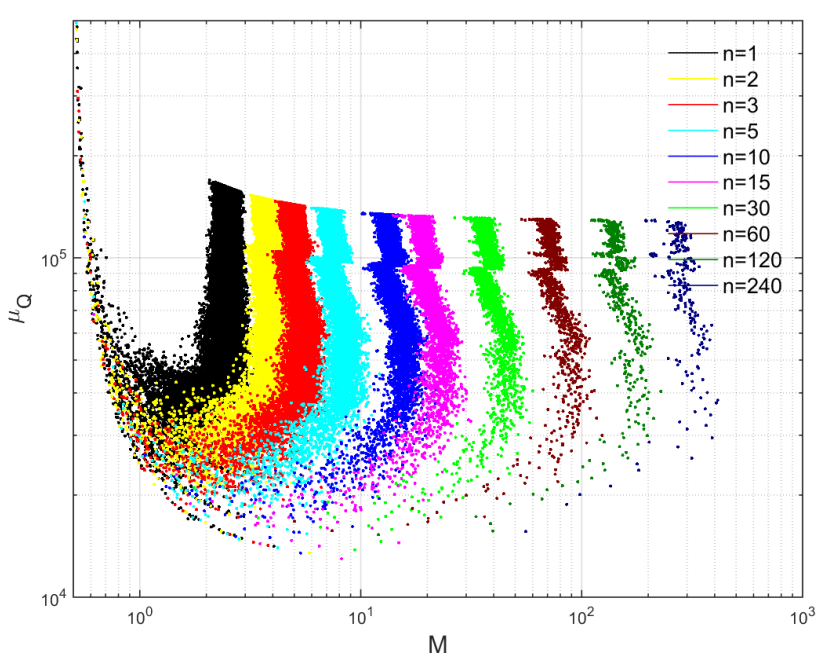

(a)

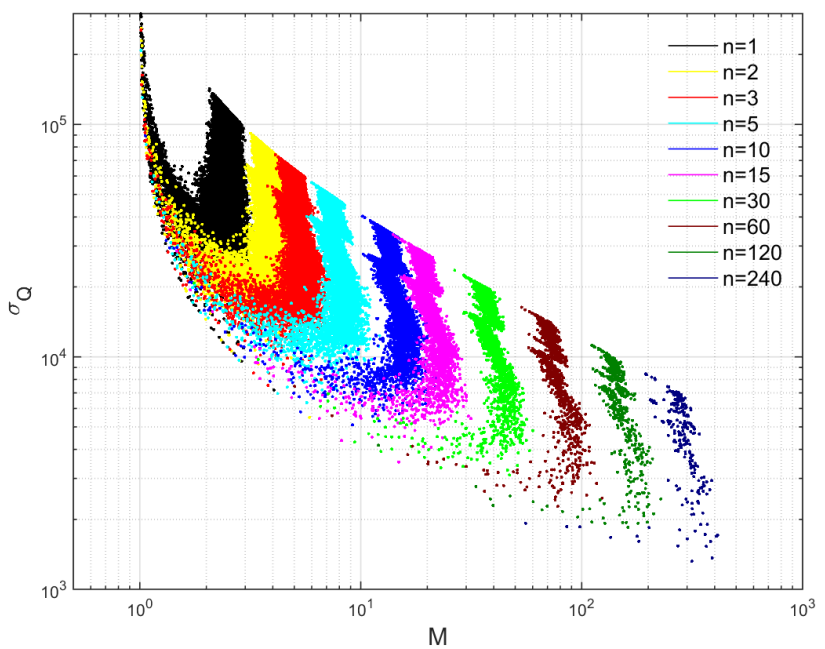

(c)

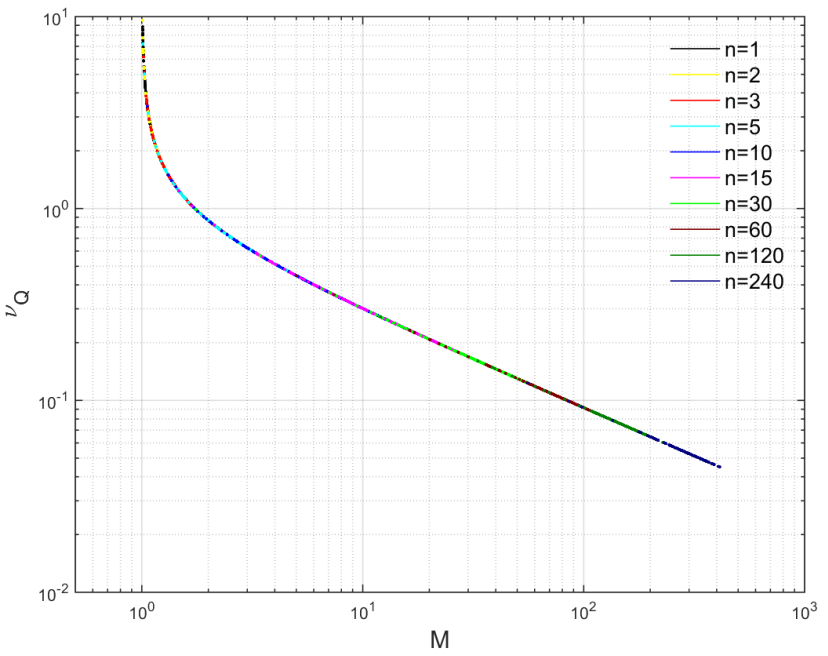

(e)

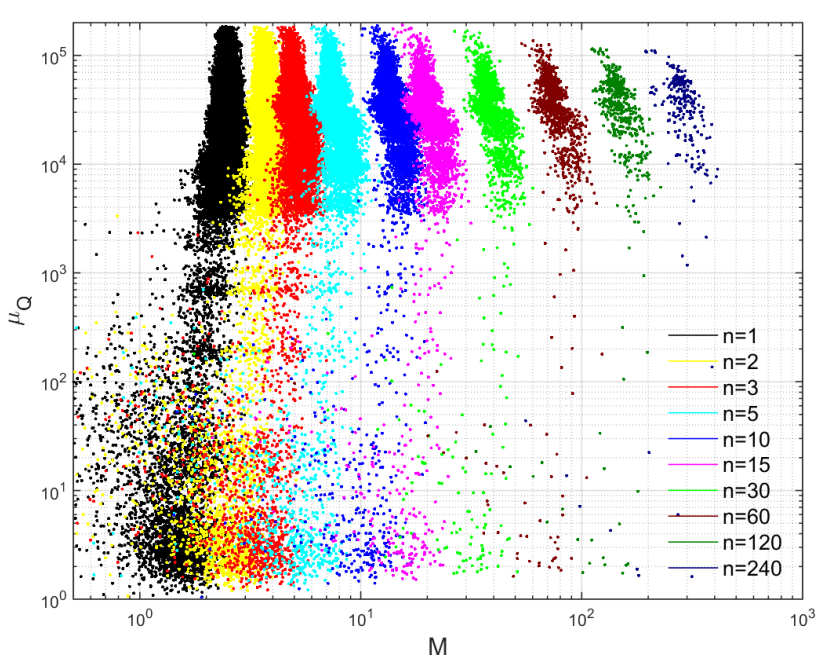

(b)

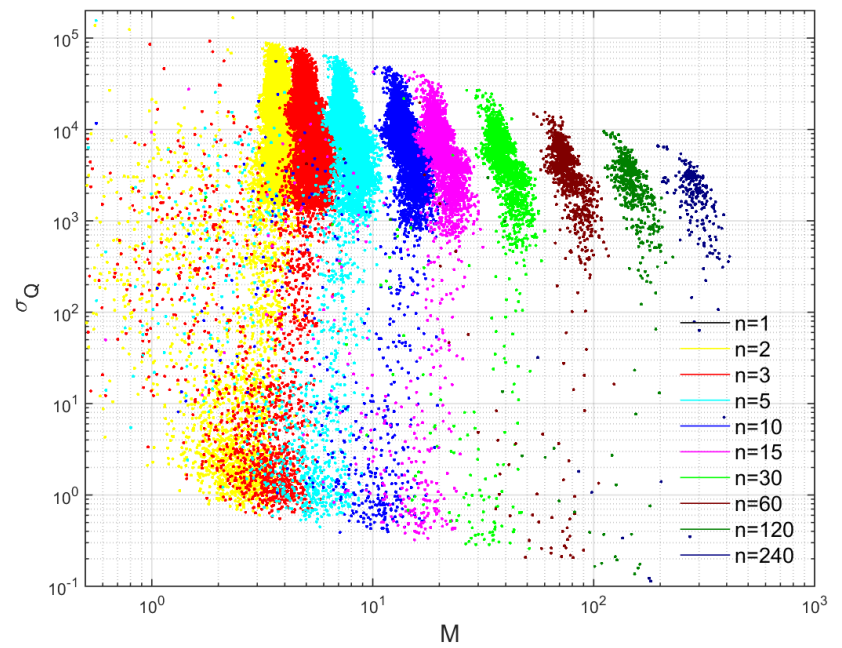

(d)

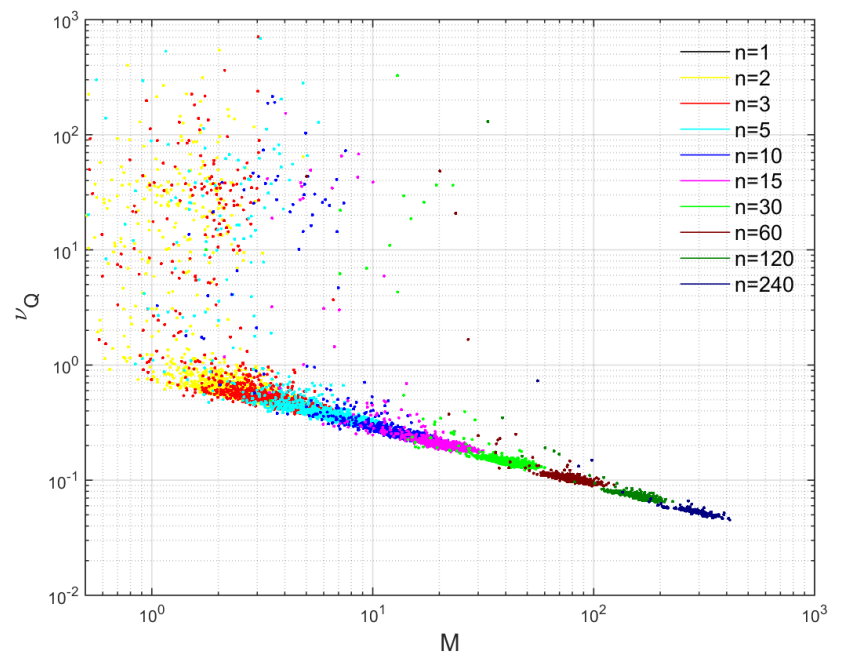

(f)

Fig. S12: Semi-analytic (left) and fully empirical (right) sample statistics of $Q$ as a function of $M$ from 0.1 to $12 \mathrm{GHz}$ (a)(b) mean value $\mu_{Q} \equiv\langle Q\rangle$, (c)(d) standard deviation $\sigma_{Q}$, (e)(f) coefficient of variation $\nu_{Q}$ as ratios of $\sigma_{Q}$ in figures (c)(d) and $\mu_{Q}$ in figures (a)(b), respectively. Note the different vertical scales between semi-analytic and fully empirical characteristics. The fully empirical $\sigma_{Q}$ and $\nu_{Q}$ for $n=1$ are zero (off-scale). 


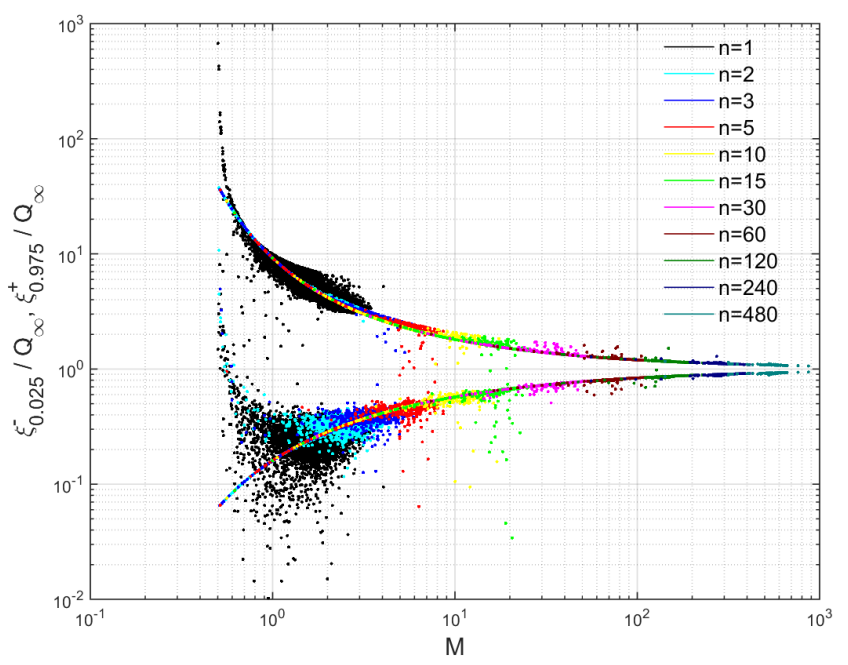

(a)

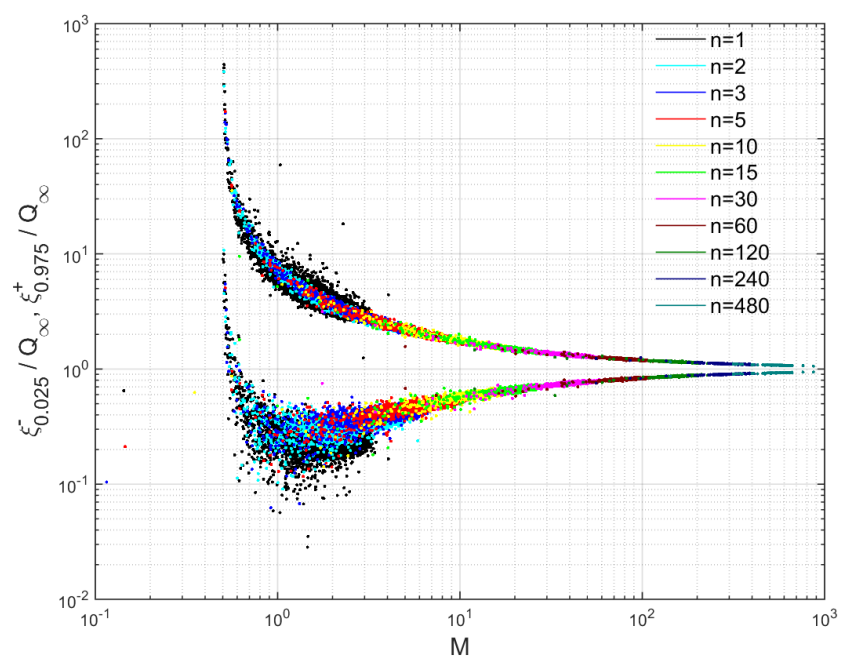

(b)

Fig. S13: Asymptotic-mean normalized (a) semi-analytic and (b) fully empirical upper and lower boundaries of $95 \%$ confidence interval of $Q$ as a function of $M$ from 0.1 to $12 \mathrm{GHz}$.

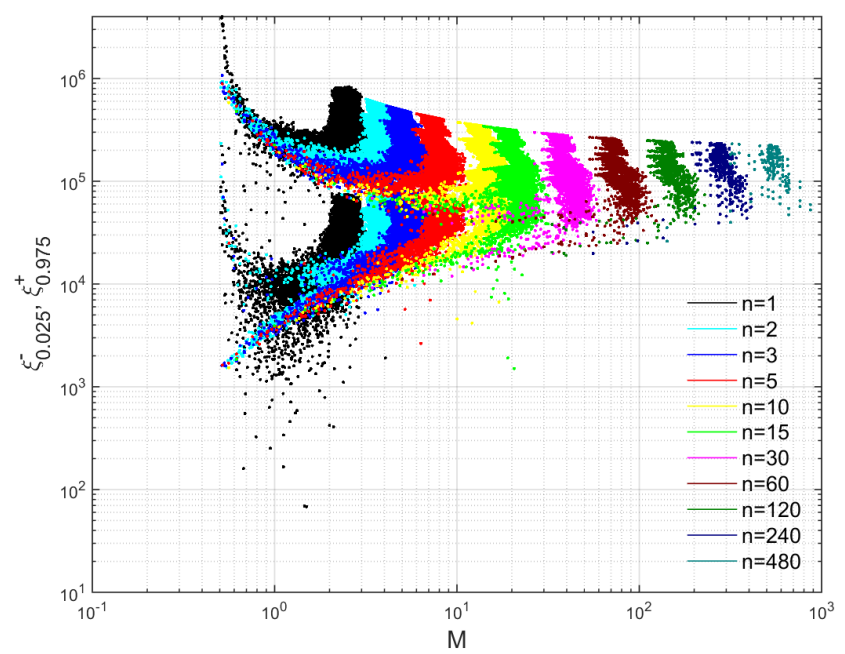

(a)

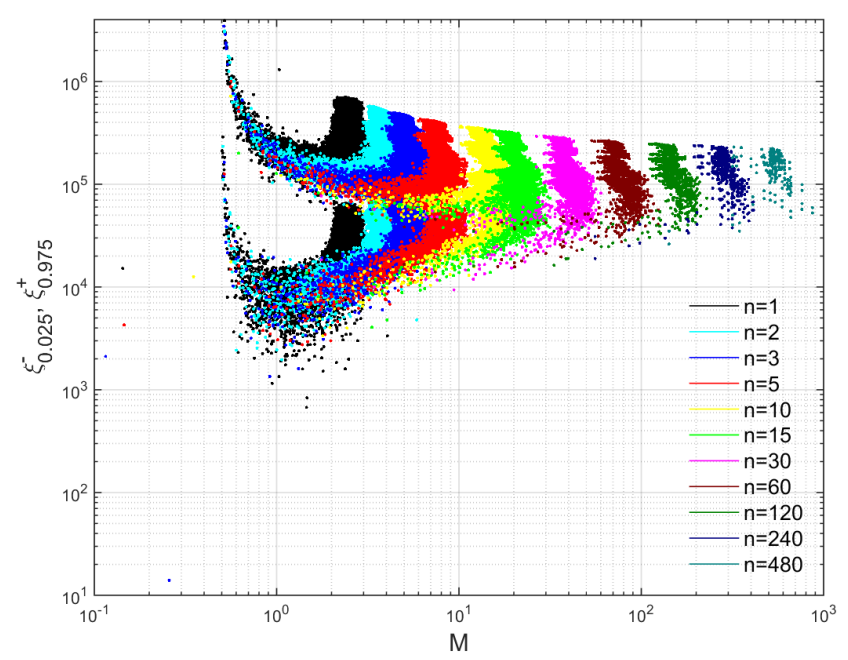

(b)

Fig. S14: Non-normalized (a) semi-analytic and (b) fully empirical upper and lower boundaries of $95 \%$ confidence interval of $Q$ as a function of $M$ from 0.1 to $12 \mathrm{GHz}$. 
SUPPLEMENTARY DATA II: $f$-CHARACTERISTICS 


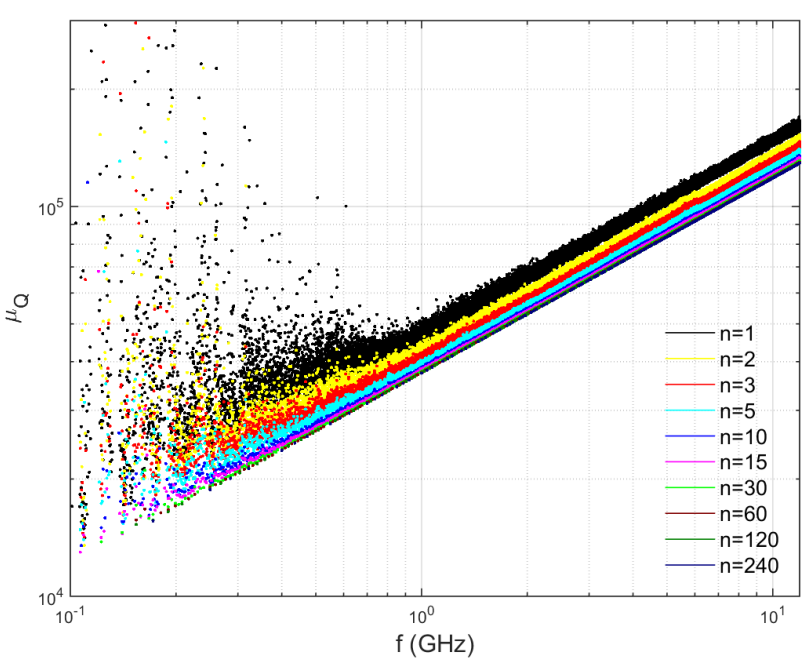

(a)

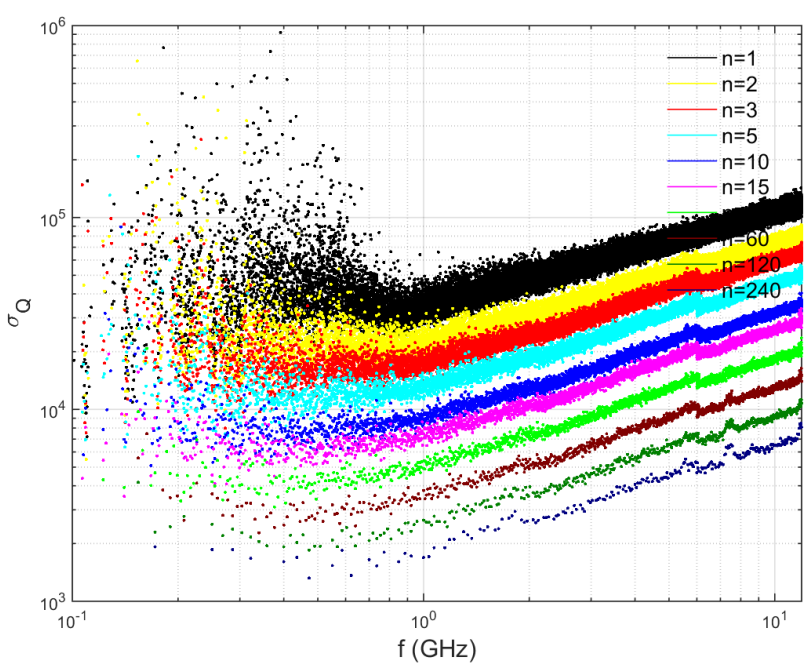

(c)

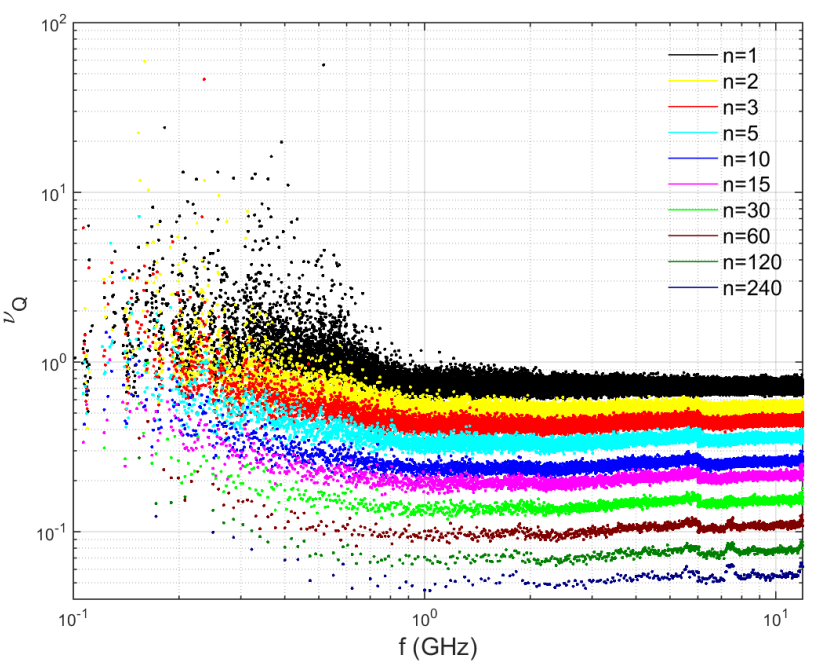

(e)

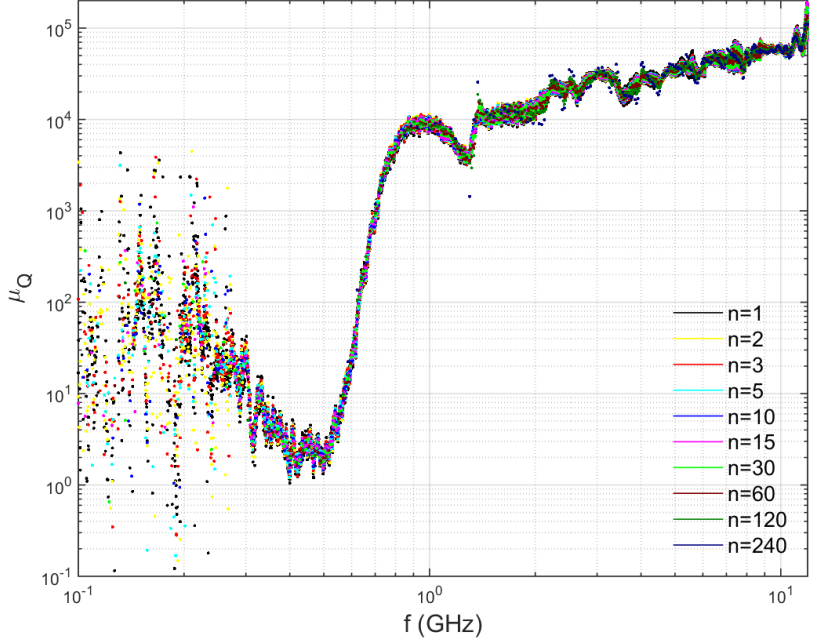

(b)

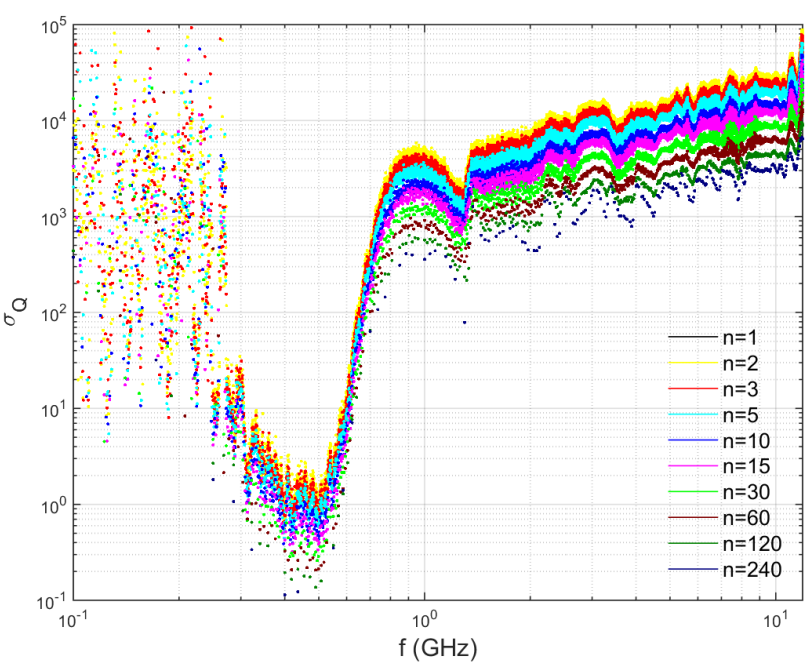

(d)

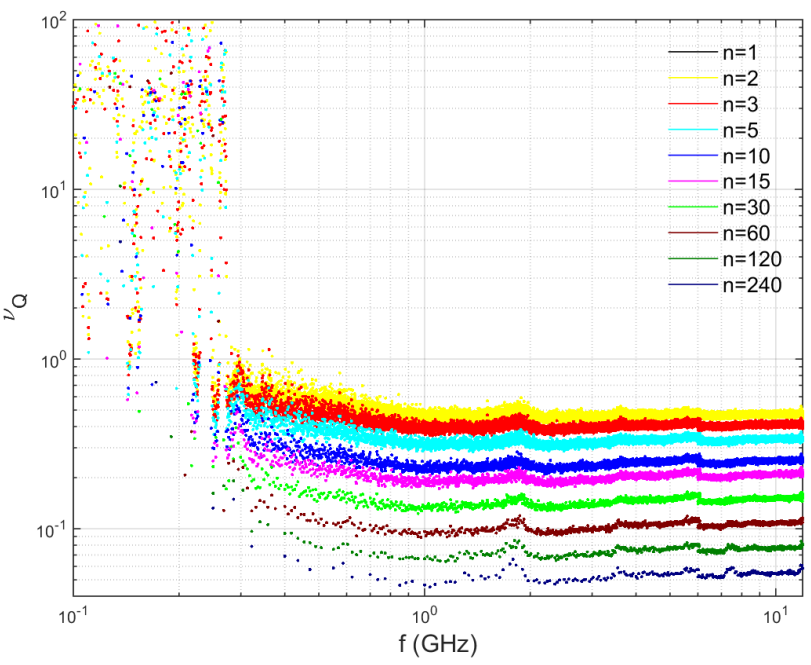

(f)

Fig. S15: Semi-analytic (left) and fully empirical (right) sample statistics of $Q$ as a function of $f$ from 0.1 to $12 \mathrm{GHz}$ : (a)(b) mean value $\mu_{Q} \equiv\langle Q\rangle$, (c)(d) standard deviation $\sigma_{Q}$, (e)(f) coefficient of variation $\nu_{Q}$. Note the different vertical scales between semi-analytic and fully empirical characteristics. The fully empirical $\sigma_{Q}$ and $\nu_{Q}$ for $n=1$ are zero (off-scale). 


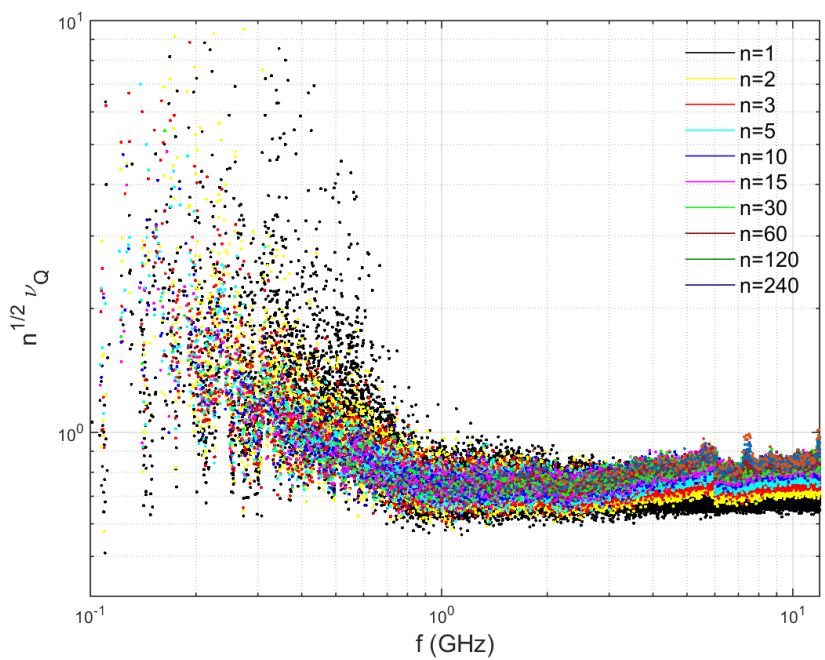

(a)

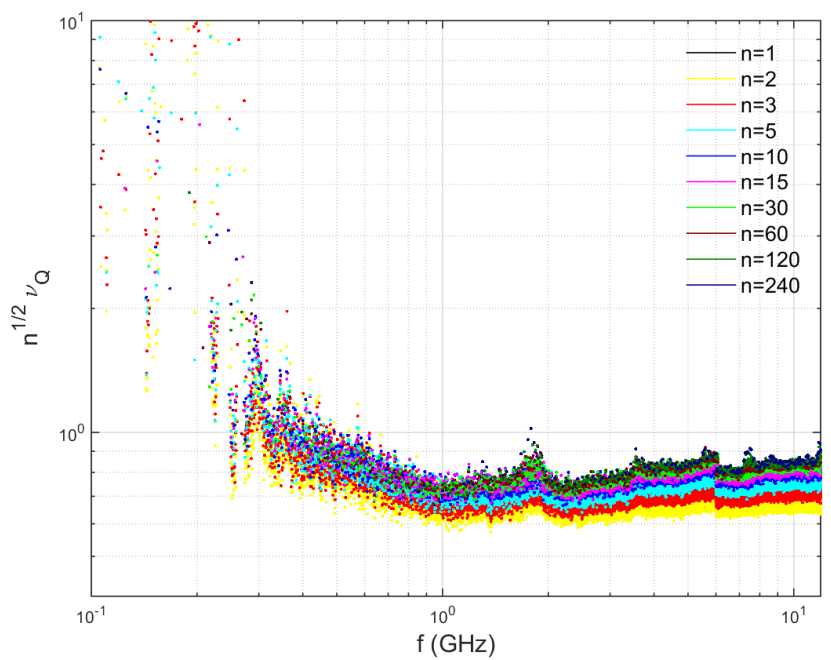

(b)

Fig. S16: (a) Semi-analytic and (b) empirical renormalized coefficient of variation $\sqrt{n} \nu_{Q}$ as a function of $f$ from 0.1 to $12 \mathrm{GHz}$. The fully empirical $\sqrt{n} \nu_{Q}$ for $n=1$ are zero (off-scale).

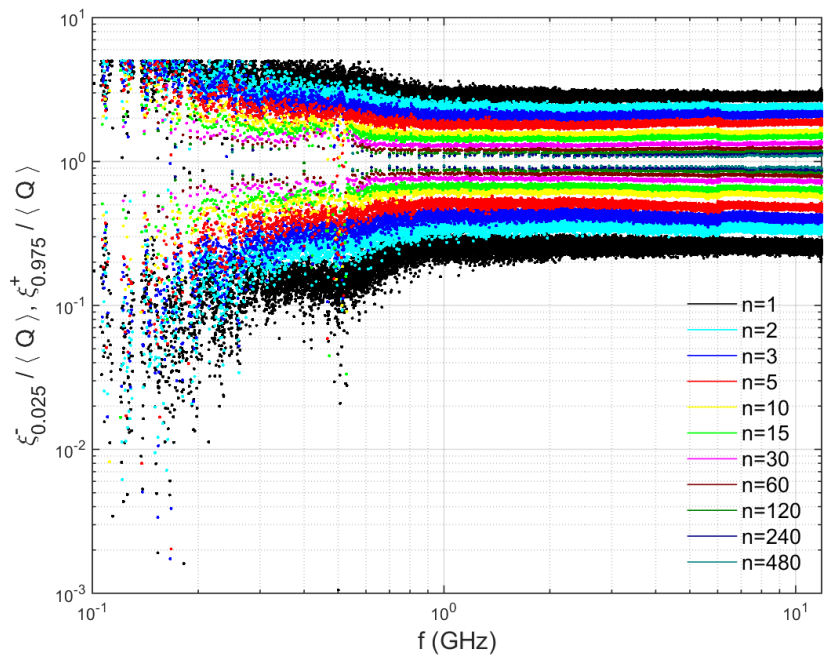

(a)

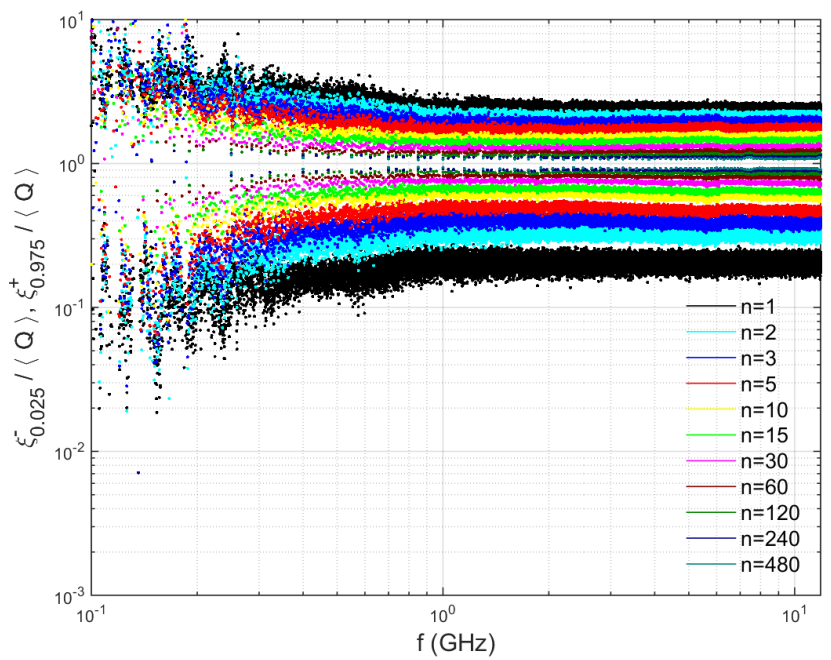

(b)

Fig. S17: Mean-normalized (a) semi-analytic and (b) fully empirical upper and lower boundaries of $95 \%$ confidence interval of $Q$ as a function of $f$ from 0.1 to $12 \mathrm{GHz}$. 


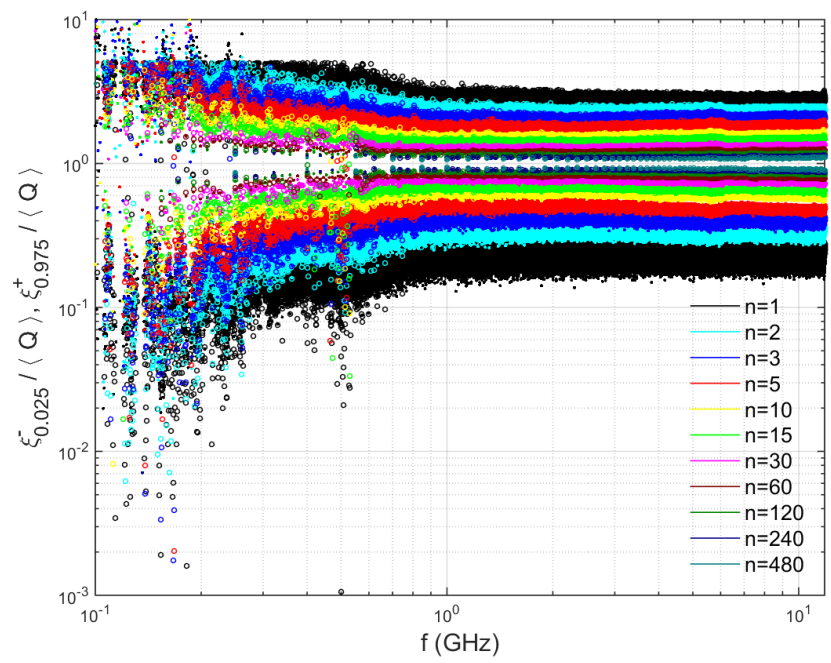

Fig. S18: Mean-normalized semi-analytic (circles) and fully empirical (dots) upper and lower boundaries of $95 \%$ confidence interval of $Q$ as a function of $f$ from 0.1 to $12 \mathrm{GHz}$.

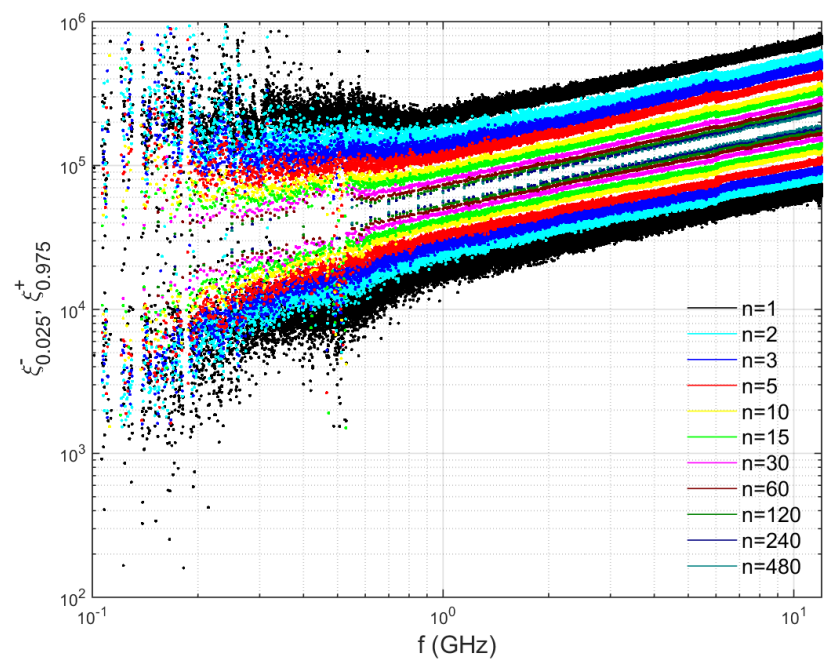

(a)

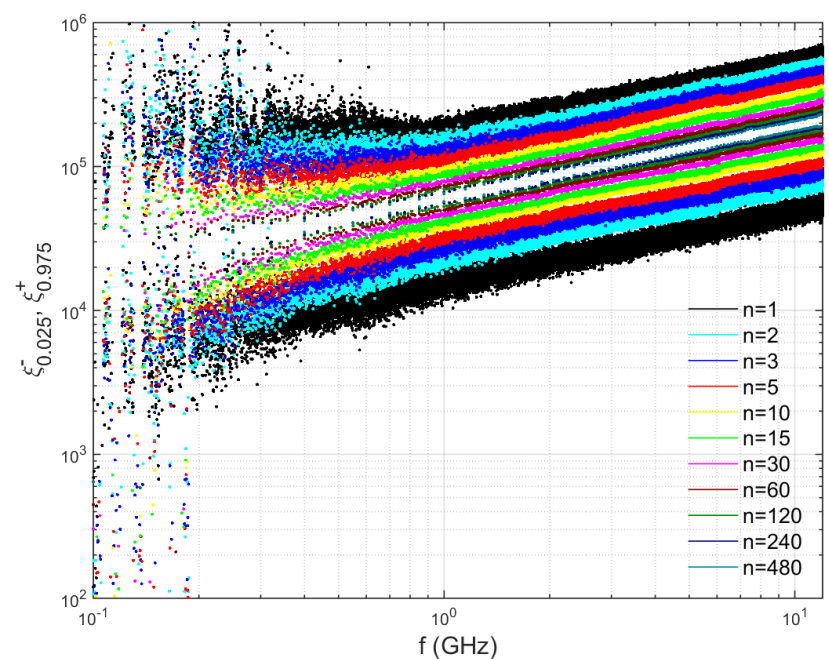

(b)

Fig. S19: Non-normalized (a) semi-analytic and (b) fully empirical upper and lower boundaries of $95 \%$ confidence interval of $Q$ as a function of $f$ from 0.1 to $12 \mathrm{GHz}$. 